\title{
Zur Theorie der zweifach unendlichen Zahlenfolgen.*)
}

\author{
Von
}

Arfred Prinasherm in München.

In einer früher publicirten Arbeit über „Elementare Theorie der unendlichen Doppelreihen"***) habe ich einige Hauptsätze über die Grenzwerthe zweifach unendlicher Zahlenfolgen zusammengestellt, soweit sie mir für den damaligen $\mathrm{Zweck}$ erforderlich schienen. Nun scheint mir aber die, meines Wissens, in der Literatur sonst nicht ausdrücklich behandelte Theorie dieser Zahlenfolgen eine weit über die erwähnte specielle Anwendung hinausreichende, principielle Bedeutung zu besitzen und darf insbesondere für die gesammte Lehre von den Functionen mehrerer Variablen als fundamental gelten: bildet doch die Zurückfuhrung sogenannter stetiger Grenzübergänge auf Grenzwerthe abzähtbarer Zahlenmengen eine der wesentlichsten Grundlagen für die moderne Verschärfung functionenthe0retischer Definitionen und Beweise. Aus diesem Grunde durfte vielleicht die folgende ausführlichere Darstellung gewisser Grenzwerth-Eigenschaften der zweifach-unendlichen Zahlenfolgen einiges Interesse beanspruchen, zumal dieselbe verschiedene nicht unerhebliche Verallgemeinerungen und Vervollständigungen der früher mitgetheilten Hauptsätze enthält. Auch möchte ich auf die hierbei sich ergebende methodisch consequente und

*) Der folgende Aufsatz ist im wesentlichen einem Abschnitte meiner für den Druck bestimmten Vorlesungen über unendliche Reihen und analytische Functionen entnommen. Da es mir in Folge von Berufsgerchaften wnd anderen nothwendigen Arbeiten bisher nicht møglich war, das betreffende Buch druckfertig zu machen, und ich andererseits von Herrn F. London erfahren habe, dass er, durch meine unten citirte Arbeit ũber Doppelréizen angeregt, eine Abhandlang ther den vorliegenden Gegenstand bei der Redaction der Mathematischen Annalen eingereicht habe, so bin ich der letzteren zu Danke verpflichtet, dass sie anch mir Gelegenheit giebt, die folgenden, in der Hauptsache aus dem Jahre 1896 stammenden Untersuchungen an dieser Stelle zu veroffentlichen. Im übrigen möchte ich nur noch ansärücklich hervor. heben, dess die Arbeit des Herrn London, trotz ihres Zusammenhanges mit mëinem oben erwăhnten friiheren Aufsatze, vollig unabhingig von den hier mitgetheilten all gemeineren. Untersachougen entstanden ist.

**) Minch...Sitz.:Ber:-Bd: 27 (1887), p. 101. 
didaktisch äusserst zweckmässige*) Einführungsart der gleichmässigen und ungleichmässigen Convergenz einigen Werth legen. Der fragliche Fundamental-Begriff erscheint hier thatsächlich in seiner einfachsten Grundform, und der Zusammenhang, in welchem er auftritt, führt zugleich zu einer, soviel ich weiss, bisher nicht bemerkten nützlichen Erweiterung, die sich auch für gewisse functionentheoretische Untersuchungen als fruchtbar erweisen dürfte. Auf Grund dieses erweiterten Begriffes lassen sich insbesondere die nothwendigen und hinreichenden Bedingungen für die Existenz von $\lim _{\mu, v=\infty} a_{\mu}^{(v)}$ bei vorausgesetzter Existenz von $\lim _{\nu=\infty} \varlimsup_{\mu=\infty} a_{\mu}^{(\nu)}$ oder $\left.\lim _{\mu=\infty} \varlimsup_{v=\infty} a_{\mu}^{(v)} * * *\right)$

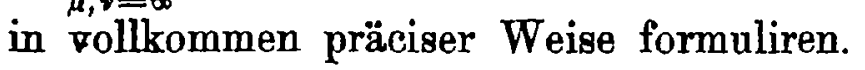

\section{$\S 1$.}

Grenzwerthe convergenter und eigentlich divergenter Doppelfolgen. Monotone Doppelfolgen.

1. Als Doppelfolge werde im folgenden jede zweifach unendliche Folge reeller Zablen $a_{\mu}^{(v)}(\mu=0,1,2, \cdots \nu=0,1,2, \cdots)$ bezeichnet. Wir denken uns dieselbe allemal in der Form des Schema's;

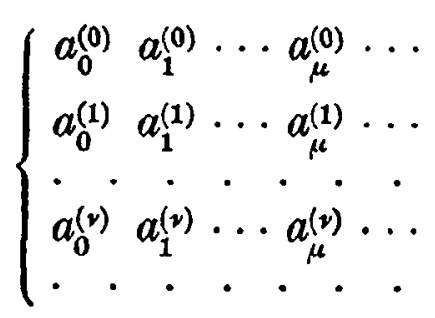

angeordnet; dabei markirt also der obere Index $v$ die Zeile, der untere Index $\mu$ die Colonne, welcher der Term $a_{\mu}^{(v)}$ angehört. Wir sagen, die Doppelfolge $a_{\mu}^{(v)}$ sei convergent und besitze für $\lim \mu=\infty, \lim \nu=\infty$ (ausführlicher: wenn $\mu, \nu$ unabhängig von einander und gleichzeitig ins Unendliche wachsen) den endlichen Grenzwerth $A$, in Zeichen:

$$
\lim _{\mu, \nu=\infty} a_{\mu}^{(\nu)}=A \text {, }
$$

wenn zu jedem (beliebig kleinen) $\varepsilon>0$ zwei natürliche Zahlen $n_{1}, n_{q}$ existiren, sodass:

$$
\left.\left|a_{. \mu}^{(\nu)}-A\right| \leqq \varepsilon \quad \text { für: } \mu \geqq n_{1}, \nu \geqq n_{\mathrm{g}} \cdot{ }^{* * *}\right)
$$

*) Mit Hülfe sehr einfacher Zahlen-Schemata (vgl. \& 3, Beisp. 1) und 3)) kann man diesen, bei der sonst üblichen Einführungsart dem Anfänger meist ausserst schwierig erscheinenden und dennoch, nach meinem Dafürhalten, selbst in ElementarPorlesungen kaum mehr zu entbehrenden Begriff, förmlich ad oculos demonstrixen.

*) Ueber den Sinn dieser Bezeichnungsweise vgl. p. 302, Fussnote.

**) Ich gebe der Formulirung: $\left|a_{\mu}^{(\nu)}-A\right| \leqq \varepsilon$ vor der zumeist üblichen: $\left|a_{\mu}^{(v)}-A\right|<\varepsilon$ den Vorzug, weil sie bei der Willkürlichkeit von $\varepsilon$ schliesalich genau 
Diese Bedingung lässt sich ohne Beschränkung der Allgemeinheit auch durch die folgende ersetzen:

$$
\left.\left|a_{\mu}^{(\nu)}-A\right| \leqq \varepsilon \quad \text { für: } \begin{array}{c}
\mu \\
\nu
\end{array}\right\} \geqq n .
$$

Denn die letztere ist einerseits als specieller Fall in (2a) enthalten, nämlich wenn $n_{1}=n_{2}$; andererseits kann man, wenn $n_{1} \gtrless n_{2}$, die Bedingung $(2 a)$ in $(2 b)$ überführen, indem es freisteht, die kleinere der beiden Zahlen $n_{1}, n_{2}$ durch die grössere zu ersetzen.

Die Doppelfolge heisst eigentlich divergent, ihr Grenzwerth $+\infty$ bezw.: $-\infty$, in Zeichen:

$$
\lim _{\mu, \nu=\infty} a_{\mu}^{(\nu)}=+\infty \quad \text { bezw.: }=-\infty,
$$

wenn zu jedem (beliebig grossen) $G>0$ zwei natürliche Zahlen $n_{1}, n_{2}$ existiren, sodass

$$
a_{\mu}^{(\nu)}>G \text { bezw. }<-G \text { für: } \mu \bar{\Sigma} n_{1}, \nu \bar{\nu} n_{2} .
$$

Dabei ist es wiederum gestattet, diese Bedingung durch die folgende $z u$ ersetzen:

$$
\left.a_{\mu}^{(\nu)}>G \text { bezw. }<-G \text { für: } \begin{array}{l}
\mu \\
\nu
\end{array}\right\} \geqq n .
$$

Die beiden durch Gl. (2) und (3) charakterisirten Fälle der Convergenz und eigentlichen Divergenz sollen gelegentlich auch durch den Ausdruck: zusammengefasst werden, dass der Limes der Doppelfolge oder auch der Doppel-Limes $\lim _{\mu, \nu=\infty} a_{\mu}^{(\nu)}$ existire.

2. Die nothwendige und hinreichende Bedingung für die Convergenz der Doppelfolge kann auch so formulirt werden, dass sie nur die $a_{\mu}^{(v)}$, nicht aber den betreffenden Grenzwerth $A$ enthält. Man kann ihr jede der folgenden drei Formen geben, welche trotz ihrer äusseren Verschiedenheit dieselbe Tragweite besitzen:

$$
\begin{array}{ll}
\left|a_{\mu+\rho}^{(\nu+\sigma)}-a_{\mu}^{(\nu)}\right| \leqq \varepsilon \text { für: } & \begin{cases}\mu \geqq n_{1} & \varrho=0,1,2, \cdots \\
\nu \geqq n_{8} & \sigma=0,1,2, \cdots\end{cases} \\
\left|a_{\nu+\rho}^{(\nu+\sigma)}-a_{\nu}^{(\nu)}\right| \leqq \varepsilon \text { für: } & \begin{cases}\nu \geqq n & \varrho=0,1,2, \cdots \\
\nu=0,1,2, \cdots & \sigma\end{cases} \\
\left.\left|a_{\mu}^{(\nu)}-a_{n}^{(n)}\right| \leqq \varepsilon \text { für: } \begin{array}{l}
\mu \\
\nu
\end{array}\right\} \geqq n . &
\end{array}
$$

Man erkennt zunächst, dass (4b) und (4c) durch successive Specialisirung aus (4a) hervorgehen. Es genügt daher, nachzuweisen, dass für die Con-

dieselbe Tragweite besitat, wie diese letztere, während andererseits ihre (aus irgendwelchen Voraussetzungen zu folgernde) Existenz gewöhnlich etwas kïrzer erwiesen werden kann, ale diejenige von: $\left|a_{\mu}^{(\nu)}-A\right|<\varepsilon$. 
A. Pravashent.

vergenz der Doppelfolge (4a) eine nothwendige, (4c) eine hinreichende $\mathrm{Be}$ dingung darstellt.

Ist nun zunächst die Folge convergent, $A$ ihr Grenzwerth, so kann man nach (2a) $n_{1}, n_{2}$ so fixiren, dass:

$$
\left|a_{\mu}^{(v)}-A\right| \leqq \frac{\varepsilon}{2} \quad \text { für: } \mu \geqq n_{1}, \nu \geqq n_{2}
$$

und somit auch:

$$
\left|a_{\mu+\rho}^{(\nu+\sigma)}-A\right| \leqq \frac{\varepsilon}{2} \quad \text { für: } \begin{cases}\mu \geqq n_{1} & \varrho=0,1,2, \cdots \\ \nu \geqq n_{2} & \sigma=0,1,2, \cdots,\end{cases}
$$

sodass durch Combination dieser beiden Ungleichungen unmittelbar die Bedingung (4a) (und somit auch (4b), (4c)) als nothwendig für die Convergenz resultirt.

Besteht andererseits die Bedingung (4c), so kann man zunächst $m$ so fixiren, dass:

$$
\left.\left|a_{\mu}^{(v)}-a_{m}^{(m)}\right| \leqq \frac{\varepsilon}{3} \quad \text { für: } \quad \begin{array}{c}
\mu \\
\nu
\end{array}\right\} \geqq m,
$$

und man hat daher speciell für $\mu=\nu$ :

$$
\left|a_{\nu}^{(\nu)}-a_{m}^{(m)}\right| \leqq \frac{\varepsilon}{3} \quad \text { für: } \quad \nu \geqq m .
$$

Die letztere Ungleichung besagt aber, dass die einfach unenaliche Folge $\left(a_{v}^{(v)}\right)$ convergent und somit eine bestimmte Zahl

$$
A=\lim _{v=\infty} a_{v}^{(v)}
$$

existirt. Man kann daher ein $n$ so fixiren, dass:

$$
\left|a_{v}^{(\nu)}-A\right| \leqq \frac{\varepsilon}{3} \quad \text { für: } \quad \nu \geqq n,
$$

wobei es von vornherein freisteht $n \geqq m$ anzunehmen. Sodann wird aber

also:

$$
a_{\mu}^{(v)}-A=a_{\mu}^{(\nu)}-a_{m}^{(m)}+\left(a_{m}^{(m)}-a_{n}^{(n)}\right)+\left(a_{n}^{(n)}-A\right),
$$

$$
\left|a_{\mu}^{(v)}-A\right| \leqq\left|a_{\mu}^{(v)}-a_{m}^{(m)}\right|+\left|a_{n}^{(n)}-a_{m}^{(m)}\right|+\left|a_{n}^{(n)}-A\right|
$$

d. h. schliesslich mit Benützung von Ungl. (5), (6), (7):

$$
\left.\left|a_{\mu}^{(\nu)}-A\right| \leqq \varepsilon \text { für: } \cdot \begin{array}{l}
\mu \\
\nu
\end{array}\right\} \geqq n,
$$

woraus nach $(2 \mathrm{~b})$ die Convergenz der Doppelfolge gegen den Grenzwerth $A\left(=\lim _{\nu=\infty} a_{v}^{(\nu)}\right)$ resultirt.

3. Die Doppelfolge $\left(a_{\mu}^{(v)}\right)$ heisst monoton und zwar niemals ab- bezw. niemals zunehmend, wenn durchweg:

$$
a_{\mu+\rho}^{(\nu+\sigma)}-a_{\mu}^{(\nu)} \geqq 0 \quad \text { bezw. } \quad a_{\mu+\rho}^{(\nu+\sigma)}-a_{\mu}^{(\nu)} \leqq 0
$$


$(\varrho=0,1,2, \cdots, \sigma=0,1,2, \cdots)$. Für solche Doppelfolgen gilt zunächst der Satz:

Bleiben die $\left|a_{\mu}^{(v)}\right|$ unter einer Zahl $g$, so ist die monotone Doppelfolge $\left|a_{\mu}^{(v)}\right|$ allemal convergent.

Beweis*). Es sei etwa die fragliche Folge $\left(a_{\mu}^{(\nu)}\right)$ eine niemals abnehmende, sodass also die erste der Ungleichungen (8) als gültig vorausgesetzt wird. Angenommen nun, die Folge sei nicht convergent, so müsste mit Rücksicht auf die Convergenzbedingung (4a), wie gross auch $\mu, \nu$ angenommen werden, zu dem Term $a_{\mu}^{(v)}$ ein anderer $a_{\mu+e}^{(n+\sigma)}$ existiren, sodass.

$$
\left|a_{\mu+\rho}^{(\nu+\sigma)}-a_{\mu}^{(\nu)}\right|=a_{\mu+\rho}^{(\nu+\sigma)}-a_{\mu}^{(\nu)}>\varepsilon
$$

wo $\varepsilon$ eine möglicherweise sehr kleine, aber bestimmte positive Zahl bedeutet. Man könnte darnach aus der Doppelfolge $\left(a_{\mu}^{(v)}\right)$ eine unbegrenst fortsetzbare Folge ron Termen:

herausheben, sodass:

$$
a_{m}^{(n)}, a_{m+\varrho_{1}}^{\left(n+\sigma_{1}\right)}, \cdots a_{m+\rho_{x}}^{\left(n+\sigma_{x}\right)}, \cdots
$$

$$
\begin{aligned}
& a_{m+\rho_{2}}^{\left(n+\sigma_{1}\right)}-a_{m}^{(n)}>\varepsilon, \\
& a_{m+\rho_{2}}^{\left(n+\sigma_{2}\right)}-a_{m+\rho_{2}}^{\left(n+\sigma_{1}\right)}>\varepsilon, \\
& \cdot \cdot \cdot \cdot \cdot \cdot \cdot \cdot \cdot \cdot \cdot \cdot \cdot \cdot \cdot \cdot \sigma_{m+\rho_{x-1}}^{\left(n+\sigma_{x}-1\right)}>\varepsilon \\
& a_{m+\rho_{x}}^{\left(n+\sigma_{x}\right)}-a^{(n)}
\end{aligned}
$$

und daher für jedes noch so grosse $n$ :

$$
a_{m+\rho_{x}}^{\left(n+\sigma_{x}\right)}-a_{m}^{(n)}>x \varepsilon \text { d. h. } a_{m+\rho_{x}}^{\left(n+\sigma_{x}\right)}>a_{m}^{(n)}+x \varepsilon,
$$

was der Voraussetzung $\left|a_{\mu}^{(v)}\right|<g$ widersprechen wird. Somit muss die niemals abnehmende Doppelfolge $\left(a_{\mu}^{(v)}\right)$ unter der gemachten Voraussetzung convergiren.

Das analoge Resultat ergiebt sich sodann ohne weiteres für eine niemals aunehmende Doppelfolge $\left(a_{\mu}^{(v)}\right)$, wenn man beachtet, dass in diesem Falle die Doppelfolge $\left(-a_{\mu}^{(v)}\right)$ eine riemals ábnehmende und

ist.

$$
\lim _{\mu, v=\infty}\left(-a_{\mu}^{(\nu)}\right)=-\lim _{\mu, \nu=\infty} a_{\mu}^{(v)}
$$

4. Bleiben die $\left|a_{\mu}^{(v)}\right|$ einer monotonen Doppelfolge nicht unter einer festen Grenze, giebt es also, wie gross man auch $G>0$ annehmen möge, stets. Terme $a_{\mu}^{(v)}$, für welche $\left|a_{\mu}^{(v)}\right|>G$, so existiren nur die folgenden beiden Möglichkéeiten:

*) Vgl. auch p. 301, Fussnote. 
Entweder die Doppelfolge $\left(a_{\mu}^{(v)}\right)$ ist eine niemals abnehmende. Dann müssen die $a_{\mu}^{(\nu)}$, wenn ihr absoluter Betrag schliesslich jedes beliebige $\boldsymbol{G}$ übersteigen soll, für hinlänglich grosse $\mu, \nu$ durchweg positiv werden, sodass also nicht nur $\left|a_{\mu}^{(\nu)}\right|>G$, sondern $a_{\mu}^{(\nu)}>G$ wird, d. h. man hat in diesem Falle:

$$
\lim _{\mu, \nu=\infty} a_{\mu}^{(\nu)}=+\infty
$$

Oder die Doppelfolge $\left(a_{\mu}^{(\nu)}\right)$ ist eine niemals zunehmende. Dann bilden wiederum die Terme $-a_{\mu}^{(v)}$ eine niemals abnehmende Folge, für welche. $\left|-a_{\mu}^{(v)}\right|$ beliebig gross wird. Man hat daher zunächst:

und daher schliesslich:

$$
\lim _{\mu, \nu=\infty}\left(-a_{\mu}^{(\nu)}\right)=+\infty
$$

$$
\lim _{\mu, v=\infty} a_{\mu}^{(v)}=-\infty
$$

Da hiernach für monotone Doppelfolgen $\lim _{\mu, \nu=\infty} a_{\mu}^{(\nu)}$ als endlich oder unendlich gross stets existirt und somit auch durch $\lim _{v=\infty} a_{v}^{(v)}$ ersetzt werden kann, so gilt auch der folgende Satz:

Eine monotone Doppelfolge $\left(a_{\mu}^{(v)}\right)$ ist entweder convergent oder eigentlich divergent; sie convergirt oder divergirt, je nachdem $\lim _{v=\infty} a_{v}^{(v)}$ endlich oder unendlich ausfällt und man hat: $\lim _{\mu, \nu=\infty} a_{\mu}^{(\nu)}=\lim _{\nu=\infty} a_{\nu}^{(\nu)}$.

\section{$\S 2$.}

Ưneigentlich divergente Doppelfoligen. - Unterer und oberer Limes (Unbestimmtheitsgrenzen) der Doppelfolge.

1. Jede Doppelfolge, welche weder convergirt, noch eigentlich divergint, soll als uneigentich divergent bezeichnet werden. Zur genaueren Charakterisirung des Verháltens, welches den Termen $a_{\mu}^{(v)}$ einer solchen Doppelfolge für $\lim \mu=\infty$, lim $\nu=\infty$ zukommt, stellen wir die folgende Betrachtung an:

Es sei $\dot{a}_{\mu}^{(\nu)}(\mu=0,1,2, \ldots ; \nu=0,1,2, \ldots)$ das allgemeine Glied eìner beliebigen ( $\mathrm{d}$. h. eventuell auch convergenten oder eigentlich divergentén) Doppelfolge. Die Gesammtheit der in dieser tooppélfolge enthaltenen Terme werde (mit Rücksicht auf das Anfangsglied $\left.a_{0}^{(0)}\right)$ mit $\left[a_{\mu}^{(\nu)}\right]_{0}^{0}$ be zeichnet; und analog bezeichne $\left[a_{\mu}^{(v)}\right]_{m}^{n}$ die Gesammtheit derjenigen Terme, 
welche nach Weglassung der ersten $m$ Colonnen und $n$ Zeilen übrig bleiben, also:

$$
\begin{aligned}
& a_{m}^{(n)} \quad a_{m+1}^{(n)} \cdots a_{m+\rho}^{(n)} \cdots \\
& a_{m}^{(n+1)} a_{m+1}^{(n+1)} \cdots a_{m+\rho}^{(n+1)} \cdots \\
& a_{m}^{(n+\sigma)} a_{m+1}^{(n+\sigma)} \cdots a_{m+\rho}^{(n+\sigma)} \cdots
\end{aligned}
$$

Diese Terme $\left[a_{\mu}^{(v)}\right]_{m}^{n}$ besitzen allemal eine untere Grenze $\underline{A}_{m}^{(n)}$ und eine obere Grenze $\bar{A}_{m}^{(n)}$, wo $\underline{A}_{m}^{(n)}, \bar{A}_{m}^{(n)}$ entweder bestimmte $Z$ ahten vorstellen oder auch $\underline{A}_{m}^{(x)}=-\infty, \bar{A}_{m}^{(n)}=+\infty$ sein kann. Dabei hat man offenbar stets:

$$
\begin{gathered}
\underline{A}_{m+\rho}^{(n+o)} \geqq \underline{A}_{m}^{(n)}, \quad \bar{A}_{m+\rho}^{(n+\sigma)} \leqq \bar{A}_{m}^{(n)} \quad\left(\begin{array}{l}
\varrho=0,1,2, \cdots \\
\sigma=0,1,2, \cdots
\end{array}\right) \\
\underline{A}_{m}^{(n)} \leqq \bar{A}_{m}^{(n)} .
\end{gathered}
$$

Von den beiden einfachen Zahlenfolgen:

$$
\begin{aligned}
& \underline{A}_{0}^{(0)}, \underline{A}_{1}^{(1)}, \cdots \underline{A}_{v}^{(v)}, \cdots \\
& \bar{A}_{0}^{(0)}, \bar{A}_{1}^{(1)}, \cdots \bar{A}_{v}^{(v)}, \cdots
\end{aligned}
$$

ist also die erste niemals abnehmend, die zweite niemals zunehmend. Dabei. kann der besondere Fall eintreten, dass die Folge (11a) aus lauter Termen $-\infty$ besteht*), sodass also auch $\lim _{\nu=\infty} \underline{A}_{\nu}^{(\nu)}=-\infty$. In jedem anderen Falle muss die Folge (11a) als niemals abnehmend convergiren oder nach $+\infty$ divergiren. Man kann also schliesslich setzen:

$$
\lim _{v=\infty} \underline{A}_{v}^{(v)}=\underline{A} \text {, }
$$

A eine bestimmte Zahl oder ein $\infty$ mit bestimmtem Vorzeichen vorstellt. Analog kann die Folge (11b) ans lauter Termen $+\infty$ bestehen, in welchem Falle dann auch $\lim _{v \rightarrow \infty} \bar{A}_{v}^{(\nu)}=+\infty$. wird. Andernfalls muss sie als niemals zunehmend convergiren oder nach $-\infty$ divergiren, und man hat schliesslich

$$
\lim _{\nu=\infty} \bar{A}_{v}^{(v)}=\bar{A}
$$

*). Dieser Fall tritt ein, wenn die Doppelfolge $\left(a_{\mu}^{(v)}\right)$ nach $-\infty$ divergirt, oder renn zum mindesten zu jedem noch so grossen $G$ und $n$ immer negative Terme $a_{\mu}^{(v)}$ vorhanden sind, die numerisch über $G$ liegen, während zugleich $\mu>n,,>n$. 
wo wiederum $\bar{A}$ eine bestimmte $Z$ ahl oder $\infty$ mit bestimmtem Vorzeichen vorstellt. Zugleich folgt aus Ungl. (10), dass allemal:

$$
\underline{A} \leqq \bar{A} \text {. }
$$

Diese beiden für das Verhalten der $a_{\mu}^{(v)}$ bei $\lim \mu=\infty, \lim \nu=\infty$ als charakteristisch sich erweisenden Zahlen $\underline{A}, \bar{A}$ sollen die Haupt-Limites (Unbestimmtheitsgrenzen) der Doppelfolge $\left(a_{\mu}^{(v)}\right)$ oder kürzer schlechthin die Doppel-Limites der $a_{\mu}^{(v)}$ für $\lim \mu=\infty, \lim \nu=\infty$ heissen, speciell $\underline{A} \operatorname{der}$ untere, $\bar{A}$ der obere Doppel-Limes in Zeichen:

$$
\varliminf_{\mu, \nu=\infty} a_{\mu}^{(\nu)}=\underline{A}, \quad \varlimsup_{\mu, \nu=\infty} a_{\mu}^{(\nu)}=\vec{A} .
$$

Man bemerke zunächst noch, dass diese Zablen $\underline{A}, \bar{A}$ auch zu Stande kommen, wenn man statt der einfachen Folgen (11a), (11b) die Doppelfolgen $\left(\underline{A}_{\mu}^{(\nu)}\right),\left(\bar{A}_{\mu}^{(\nu)}\right)(\mu=0,1,2, \cdots ; \nu=0,1,2, \cdots)$ in Betracht zieht. Denn da diese letzteren nach Ungl. (5) monoton sind, so erkennt man unmittelbar mit Hiulfe des Schluss-Satzes in $\$ 1$, dass*):

$$
\lim _{\mu, \nu=\infty} \underline{A}_{\mu}^{(v)}=\lim _{\nu=\infty} \underline{A}_{\nu}^{(v)}, \quad \lim _{\mu, \nu=\infty} \vec{A}_{\mu}^{(v)}=\lim _{\nu=\infty} \vec{A}_{\nu}^{(v)} \text {. }
$$

Ferner sei noch hervorgehoben, dass wegen der Monotonie der Doppelfolgen $\left(\underline{A}_{\mu}^{(\nu)}\right) ;\left(\bar{A}_{\mu}^{(\nu)}\right)$ die Zahl $\underline{A}$ statt als $\lim _{\mu, \nu=\infty} \underline{A}_{\mu}^{(\nu)}$ auch als obere Grenze $\operatorname{der} \underline{A}_{\mu}^{(v)}$ und analog $\bar{A}$ als untere Grenze $\stackrel{\mu}{\mu}, \nu=\infty^{-(v)}$ definirt werden kann.

2. Es mögen nun zunächst $\underline{A}$ und $\bar{A}$ als endlich vorausgesetzt werden. Auf Grund der Definitionsgleichungen (12a), (12b) muss sich dann zu jedem beliebig kleinen $\varepsilon>0$ ein $n$ so fixiren lassen, dass:

(16) $\underline{A}-\varepsilon \leqq \underline{A}_{\nu}^{(\nu)} \leqq \underline{A}+\varepsilon, \quad \bar{A}-\varepsilon \leqq \bar{A}_{\nu}^{(v)} \leqq \bar{A}+\varepsilon \quad$ für $\quad v \geqq n$.

*) Es thut der Anwendbarkeit jenes. Satzes keinen Eintrag, dass unter den, $\underline{A}_{\mu}^{(\nu)}$ bezw. $\bar{A}_{\mu}^{(\nu)}$ beliebig oft der Term $-\infty$ bezw. $+\infty$ vorkommen kann, während in \$ 1 die $a_{\mu}^{(\nu)}$ durchweg als bestimmte Zahten anzusehen sind. Betrachtet man z. $\mathbf{B}$. die Folge der $\underline{A}_{\mu}^{(v)}$, so ist die Möglichkeit $\underline{A}_{m}^{(n)}=+\infty$ (wegen der Bedeutung von $\underline{A}_{m}^{(n)}$ als untere Grenze der für jedes endliche $(\mu, v)$ als endlich zu denkender Terme $\left.\left[a_{\mu}^{(v)}\right]_{m}^{n}\right)$ definitiv ausgeschlossen. Ist nun aber etwa $\underline{A}_{0}^{(0)}=-\infty$, so kann entweder nur der im Texte herrorgehobene Fall eintreten, dass alle $\underline{A}_{v}^{(v)}=-\infty$ und folglich wegen der Monotonie der $\underline{A}_{\mu}^{(\nu)}$ auch alle $\underline{A}_{\mu}^{(\nu)}=-\infty$, sodass also schliesslich:

$$
\lim _{\mu, \nu=\infty} \stackrel{A}{(\nu)}_{\mu}^{(\nu)}=\lim _{v=\infty} \underline{A}_{v}^{(\nu)}=-\infty
$$

Oder: Es giebt einmal ein endliches $\underline{A}_{n}^{(n)}$. Dann besteht aber die Doppelfolge $\left[\underline{A}_{\mu}^{(\nu)}\right]_{n}^{n}$ aus lauter endlichen Termen und gestattet somit ohne weitexes die Anwendung des fraglichen Satzes. Disis analoge gilt beżüglich der $\bar{A}_{\mu}^{(\boldsymbol{\nu})}$. 
Da nun andererseits wegen der Bedeutung der Zahlen $\underline{A}_{v}^{(v)}, \bar{A}_{v}^{(v)}$ :

$$
\left.\underline{A}_{n}^{(n)} \leqq a_{\mu}^{(\nu)} \leqq \bar{A}_{(x)}^{(n)} \quad \text { für: } \quad \begin{array}{c}
\mu \\
\nu
\end{array}\right\} \geqq n
$$

so folgt zunächst, wenn man in Ungl. (16) $\nu=n$ setzt, mit Berücksichtigung von (17):

$$
\left.\underline{A}-\varepsilon \leqq a_{\mu}^{(\nu)} \leqq \bar{A}+\varepsilon \quad \text { für: } \begin{array}{c}
\mu \\
\nu
\end{array}\right\} \geqq n .
$$

Diese Ungleichung enthält die erste Haupteigenschaft der Limites $\underline{A}, \bar{A}$, nämlich:

(I) Bei beliebig klein vorgeschriebenem $\varepsilon>0$ und hinlänglich grossem $n$ gehören alle Terme der Doppelfolge $\left[a_{\mu}^{(v)}\right]_{n}^{n}$ dem Zahlenintervalle $(\underline{A}-\varepsilon, \bar{A}+\varepsilon)$ an.

Des weiteren folgt aus der Bedeutung von $\underline{A}_{v}^{(v)}$ als untere Grenze der mit $a_{v}^{(v)}$ beginnenden Doppelfolge, dass mindestens ein Term $a_{\rho}^{(\sigma)}$ vorhanden sein muss, sodass:

$$
\left.\underline{A}_{\nu}^{(v)}-\frac{\varepsilon}{2}<\underline{A}_{\nu}^{(v)} \leqq a_{\rho}^{(\sigma)}<\underline{A}_{\nu}^{(\nu)}+\frac{\varepsilon}{2}, \text { wo: } \frac{\rho}{\sigma}\right\} \geqq \nu .
$$

Da man andererseits nach Ungl. (16) $\nu$ von vornherein so gross annehmen kann, dass:

$$
\underline{A}-\frac{\varepsilon}{2} \leqq A_{v}^{(v)} \leqq \underline{A}+\frac{\varepsilon}{2}
$$

so ergiebt sich das Bestehen der Ungleichungen:

$$
\left.\underline{A}-\varepsilon<a_{\rho}^{(\sigma)}<\underline{A}+\varepsilon \text { für: } \begin{array}{l}
\rho \\
\sigma
\end{array}\right\} \geqq \nu
$$

in dem folgenden Sinne: Wird $\varepsilon>0$ beliebig klein, $\nu$ beliebig gross vorgeschrieben, so existirt allemal mindestens ein Term $a_{\rho}^{(\sigma)}$, welcher den Bedingungen (21) genügt.

Nun bedeute $\varepsilon_{v}(\nu=0,1,2, \cdots)$ eine unbegrenzte Folge positiver Zahlen mit dem Grenzwerthe $\lim _{r=\infty} \varepsilon_{v}=0$, und es sei $a_{m_{0}}^{\left(n_{0}\right)}$ irgend ein bestimmter (auf Grund von (21) sicher vorhandener) Term, welcher der Bedingung genügt:

$$
\underline{A}-\varepsilon_{j}<a_{m_{0}}^{\left(n_{0}\right)}<\underline{A}+\varepsilon_{0} .
$$

Wird alsdann die ganze Zahl $\dot{r}_{0}>m_{0}$ und $>n_{0}$ angenommen, só resultirt aus (21) die Existenz eines Terms $a_{m_{1}}^{\left(m_{2}\right)}$. von der Beschaffenheit, dass:

$$
\left.\underline{A}-\varepsilon_{1}<a_{m_{1}}^{\left(n_{1}\right)}<\underline{A}+\varepsilon_{i} ; \text { wo: } \begin{array}{l}
m_{1} \\
n_{1}
\end{array}\right\} \geqq r_{0}, \text { also: }\left\{\begin{array}{l}
m_{1}>m_{0}, \\
n_{1}>n_{0} .
\end{array}\right.
$$


Daraus folgt in analoger Weise:

$$
\underline{A}-\varepsilon_{2}<a_{m_{2}}^{\left(n_{2}\right)}<\underline{A}+\varepsilon_{2}, \text { wo: }\left\{\begin{array}{l}
\dot{m}_{2}>m_{1}, \\
n_{2}>n_{1},
\end{array}\right.
$$

und bei Fortsetzung dieser Schlussweise:

$$
\underline{A}-\varepsilon_{\nu}<a_{m_{\nu}}^{\left(n_{\nu}\right)}<\underline{A}+\varepsilon_{\nu}, \text { wo: }\left\{\begin{array}{l}
m_{\nu}>m_{\nu-1} \\
n_{\nu}>n_{\nu-1}
\end{array}(\nu=1,2,3, \cdots) .\right.
$$

Da vollkommen analoge Beziehungen sich auch für $\bar{A}$ ergeben, so kann man als zweite Haupteigenschaft von $\underline{A}, \bar{A}$ im Anschlusse an Ungl. (21), (22) folgendes aussprechen:

(II) $Z u$ jedem beliebig klein vorgeschriebenem $\varepsilon>0$ giebt es unendlich viele, jede beliebig gross vorgeschriebene Zahl übersteigende Zahlenpaaire $(\mu ; \nu)$, sodass:

$$
\underline{A}-\varepsilon<a_{\mu}^{(\nu)}<\underline{A}+\varepsilon \quad \text { bezw. } \bar{A}-\varepsilon<a_{\mu}^{(v)}<\bar{A}+\varepsilon .
$$

Insbesondere existiren zu jeder positiven, nach 0 convergirenden Zahlenfolge $\left(\varepsilon_{v}\right)$ monoton in's Unendliche wachsende Folgen natïrlicher Zahlen $m_{v}, n_{v}$ bezw. $p_{v}, q_{v}$, sodass:

$$
\underline{A}-\varepsilon_{v}<a_{m_{v}}^{\left(n_{v}\right)}<\underline{A}+\varepsilon_{v} \quad \text { bezw. } \bar{A}-\varepsilon_{v}<a_{p_{\nu}}^{\left(q_{\nu}\right)}<\bar{A}+e_{\nu} .
$$

3. Es soll jetzt der Fall betrachtet werden, dass $\underset{\mu, \nu=\infty}{\lim } a_{\mu}^{(\nu)}=\underline{A}$ endlich, dagegen $\varlimsup_{\mu, \nu=\infty} a_{\mu}^{(\nu)}=+\infty$. Alle auf die Zahl $\underline{A}$ bezüglichen Ergebnisse bleiben alsdann unverändert, während an die Stelle der Ungleichung (18) nunmehr die folgende tritt:

$$
\left.\underline{A}-\varepsilon \leqq a_{\mu}^{(\nu)}<+\infty \quad \text { für: } \begin{array}{l}
\mu \\
v
\end{array}\right\} \geqq n,
$$

d. h.:

(Ia) Bei beliebig klein vorigeschriebenem $\varepsilon>0$ und hinlänglich gross gewähltem n gehören alle Terme der Doppel. folge $\left[a_{\mu}^{(\nu)}\right]_{n}^{n}$ dem Intervalle $(\underline{A}-\varepsilon, \infty)$ an:

Andererseits erheischt die Annahme $\varlimsup_{\mu, v=\infty} a_{\mu}^{(i)}=+\infty$, d. h. schliesslich $\lim _{\nu=\infty} \bar{A}_{v}^{(v)}=\infty$, wie in Nr. 1 bemerkt wurde, dass geradezu

$$
\bar{A}_{\nu}^{(v)}=\infty \quad(\nu=0,1,2, \cdots) .
$$

Aus der Bedeutung von $\bar{A}_{v}^{(\nu)}$ als oberer Grenze der mit $a_{v}^{(\nu)}$ beginnenden Doppelfolge ergiebt sich dann aber: wird $! G$. und $\nu$ beliebig gross vor: geschrieben, so giebt es stets Terme $a_{\varrho}^{(\sigma)}$, sodass:

$$
\left.a_{\rho}^{(\sigma)}>G \text { und zugleich: } \begin{array}{c}
\rho \\
\sigma
\end{array}\right\}>v .
$$


Bedeutet nun $G_{v}(\nu=0,1,2, \cdots)$ eine Folge positiver Zahlen mit dem Grenzwerthe $+\infty$, so hat man zunächst fur irgend ein bestimmtes Zablenpaar $p_{0}, q_{0}$ :

$$
a_{\infty}^{\left(g_{0}\right)}>G_{0}
$$

und sodann, wenn $r_{0} \geqq p_{0}$ und $\geqq q_{0}$ angenommen wird, anf Arund ron Ungl. (26):

$$
\left.a_{p_{1}}^{\left(q_{1}\right)}>G_{1} \text {, wo: } \begin{array}{c}
p_{1} \\
q_{1}
\end{array}\right\}>r_{0,} \text { also auch: }\left\{\begin{array}{l}
p_{1}>p_{0}, \\
q_{1}>q_{0}
\end{array}\right.
$$

In dieser Weise weiter fortschliessend gelangt man zu einer Beziehung von der Form:

$$
a_{\varphi_{\nu}}^{\left(q_{v}\right)}>G_{v}, \quad \text { wo: } \quad\left\{\begin{array}{l}
p_{v}>p_{r-1} \\
q_{v}>q_{r-1}
\end{array}(\nu-1,2,3, \cdots) .\right.
$$

Somit tritt hier an die Stelle der Haupteigenschaft (II), soweit wie wich suf $\varlimsup_{\mu, \nu=\infty} a_{\mu}^{(v)}$ bezieht, die folgende (s. Ungl. (26), (27)):

(III) $Z u$ jedem beliebig gross vorgeschriebenem $G>0$ giebt es unendlich viele, jede beliebig gross vorgeschriebewe Zahl übersteigende Zahlenpaare $(\mu, \nu)$, sodass:

$$
a_{\mu}^{(v)}>G \text {. }
$$

Insbesondere existiren jeder pasitiven, mach $+\infty$ divergiren den Zahlenfolge $\left(G_{v}\right)$ monotor in's Unenditicho wacheoude Folgen natiirlicher Zahlen $p_{v}, g_{n}$, sodass:

$$
a_{\rho_{v}}^{\left(\rho_{\nu}\right)}>G_{r}
$$

Es ist ohne weiteres klar, wio dieses Resultat fur den Fall:

$$
\lim _{\mu, i=\infty} a_{\mu}^{(\nu)}=-\infty, \varlimsup_{\mu, v>\infty} a_{\mu}^{(\nu)}-\bar{A} \text { (endlich) oder }-+\infty \text {, }
$$

zu modificiren ist.

4. Ist $\underline{A}=\bar{A}$ endlich, so geht Ungl. (18), wenn man $A$ fur $\underline{A}$ and $\bar{A}$ schreibt, in die folgende tuber:

$$
\left.A-\varepsilon \leqq a_{\mu}^{(\nu)} \leqq A+\varepsilon \text { d. h. }\left|a_{\mu}^{(\nu)}-A\right| \leqq \text { fur: } \begin{array}{l}
p \\
\nu
\end{array}\right\} \geq n,
$$

welche mit der Convergens-Bedingung (2a) znsammenfillt. Die Doppelfolge convergirt also in diesem Falle gegen den Grenzwerth 1, d. h. man hat:

$$
\lim _{\mu, v \rightarrow \infty} a_{\mu}^{(v)}=\lim _{\mu, v=\infty} a_{\mu}^{(v)}-\varlimsup_{\mu, v m \infty} a_{\mu}^{(v)} \text {. }
$$

Diese letztere Beziehung gilt auch noch, wenn $+\infty$ oder $-\infty$ an die Stelle der exidlichen Zahl $A$ tritt. Iot nämlich

$$
\lim _{\mu, \gamma \rightarrow \infty} a_{\mu}^{(\eta)}=\lim _{r \rightarrow \infty} \underline{A}_{r}^{(v)}-+\infty
$$


(in welchem Falle dann nach Ungl. (13) eo ipso auch $\varlimsup_{\mu, v=\infty} a_{\mu}^{(\nu)}=+\infty$ ), so kann man zu beliebig grossem $G$ ein $n$ so fixiren, dass:

$$
\underline{A}_{n}^{(n)}>G
$$

und somit, da $\underline{A}_{n}^{(n)}$ die untere Grenze der Terme $\left[a_{\mu}^{(v)}\right]_{n}^{n}$ :

$$
\left.a_{\mu}^{(\nu)}>G \quad \text { für: } \begin{array}{c}
\mu \\
\nu
\end{array}\right\} \geqq n
$$

d. h. (s. Ungl. (3b)): Die Doppelfolge divergirt nach $+\infty$.

Das entsprechende ergiebt sich dann im Falle $\varlimsup_{\mu_{2}, \nu=\infty} a_{\mu}^{(\nu)}=-\infty$.

Die convergenten und eigentlich divergenten Doppelfolgen sind also durch die Beziehung:

$$
\lim _{\mu, \nu=\infty} a_{\mu}^{(\nu)}=\varlimsup_{\mu, \nu=\infty} a_{\mu}^{(\nu)}
$$

die uneigentlich divergenten durch:

charakterisirt.

$$
\underline{\lim }_{\mu, \nu=\infty} a_{\mu}^{(\nu)}<\varlimsup_{\mu, \nu=\infty} a_{\mu}^{(\nu)}
$$

5. Man kann den vorstehenden Resultaten noch eine andere, vielleicht anschaulichere Fassung geben. Es bedeute $m_{v}, n_{v}(\nu=0,1,2, \cdots)$ je eine Folge natürlicher monoton in's Unendliche wachsender Zahlen. Alsdann soll die aus der Doppelfolge $\left(a_{\mu}^{(v)}\right)$ herausgehobene einfache Folge

$$
\left(a_{m_{v}}^{n_{v}}\right)(\nu=0,1,2, \cdots)
$$

eine ächte Theilfolge der ersteren heissen*). Dies vorausgeschickt lässt sich der Inhalt der in Nr. 2 und 3 entwickelten Beziehungen auch folgendermassen aussprechen:

*) Z. B.. $\left(a_{v}^{(v)}\right)(v=0,1,2, \cdots)=$ Folge der in der Haupt-Diagonale enthaltenen Glieder: $\left(a_{\nu+q}^{(v)}\right),\left(a_{v}^{(v+q)}\right)=$ jede zur Haupt-Diagonale parallele Folge. Ferner die beliebigen "geradlinigen!! Folgen: $\left(a_{p v+q}^{(v)}\right),\left(\alpha_{v}^{(p v+q)}\right)$, die "parabolischen“ Folgen: $\left(a_{p}^{(v)} v_{q}^{2}\right),\left(a_{v}^{\left(p v^{2}+q\right.}\right)$, etc. Die Bedeutung dieser letzteren Terminologie leuchtet ohne weiteres ein, wenn man sich in dem Schema (1) jeden einzelnen Term $a_{\mu}^{(v)}$ genau in dem Punkte mit den rochtwinkligen Coordinaten $(\mu,-v)$ concentrirt denkt. Bedeutet dann $\varphi(\nu)$ irgend eine genzzahlige Function von $\nu$, so enthalten die Folgen: $\left(a_{\varphi(v)}^{(\nu)}\right)$, $\left(a_{v}^{(\varphi(v))}\right)$ slle diejenigen Terme, deren zugeordnete: Punkte. ("Gitterprenkte") von der Curve

getroffen werden.

$$
x=\varphi(-y) \text { bezw: } y=-\varphi(x)
$$


Ist $\varliminf_{\mu, \nu=\infty} a_{\mu}^{(\nu)}=\underline{A}, \varlimsup_{\mu, \nu=\infty} a_{\mu}^{(\nu)}=\bar{A}(100 \underline{A}, \bar{A}$ endlich oder $\infty$ mit bestimmtem Vorzeichen), so existiven ächte Theilfolgen mit den Grenzwerthen $A$ und $A$ (s. Ungl. (24), (29)); dagegen giebt es keine ächte Theilfolge, welche einen kleineren Grenswerth als $\underline{A}$ oder einen grösseren als $\bar{A}$ besitet (s. Ungl. (18), (25)).

Ferner folgt für den Fall $\underline{A}=\bar{A}$ :

Ist die Doppelfolge convergent oder eigentlich divergent, so gilt das entsprechende von jeder Theilfolge, bei welcher beide Indices in's Unendliche wachsen (s. Ungl. (2a), (4a) und (3a)), insbesondere also von jeder ächten Theilfolge.

Umgekehrt:

Weiss man nur, dass jede ächte Theilfolge convergirt beswo. eigentlich divergirt, so gilt das entsprechende von der Doppelfolge selbst*).

Wenn nämlich die ächten Theilfolgen convergiren, so kann nach dem eben ausgesprochenen Satze die Doppelfolge zunächst nicht eigentlich divergiven. Sie kann aber auch nicht uneigentlich divergiven, $d$. ' $h$. verschiedene Limites $\underline{A}, \bar{A}$ besitzen. Denn alsdann könnte man zunächst aus der Doppelfolge zwei ächte Theilfolgen mit den Limites $A$ und $\bar{A}$ herausheben und aus diesen durch Vereinigung passend ausgewälter Glieder eine uneigentlich divergente ächte Theilfolge (mit den Limites $\underline{A}, \vec{A}$ ) bilden, was der Voraussetzung widerspricht. Somit muss in diesem Falle die Doppelfolge convergiren.

Das analoge gilt für den Fall, dass die ächten Theilfolgen eigentlich divergiren.

\&3.

Die Limites und iterirten Limites der Zeilen und Colonnen. Gleichmässige Convergenz, Divergenz, Begrenztheit und Unbegrenztheit der Zellen (Colonnen).

1. Wesentlich verschieden von den ächten Theilfolgen, als dem einfachsten Typus von Theilfolgen, bei welchen beide Indices schliesslich in's Unendliche wachsen, sind diejenigen Theilfolgen, bei denen nur der eine Index diese Eigenschaft besitzt und als deren einfachster Typus die Zeiten

und Colonnen:

$$
\dot{a}_{0}^{(v)} a_{1}^{(v)} \ldots a_{\mu}^{(\nu)} \ldots \quad(\nu=0,1,2, \cdots)
$$

$$
a_{\mu}^{(0)} a_{\mu}^{(1)} \cdots a_{\mu}^{(\nu)} \cdots \quad(\mu=0,1,2, \cdots)
$$

*) Hieraus konnte man auch ohne weiteres die Convergenz bezw. fignuliche. Divergenz jeder monotonen Doppelfolge erschliessen. 
des Schema's (1) erscheinen. Man bemerkt zunächst, dass das Verhalten einer beliebigen endlichen Anzahl von Zeilen und Colonnen auf die in $\$ 1,2$ näher entwickelten Limites der Doppelfolge keinerlei Einfluss übt. Denn die zur Charakterisirung dieser Limites dienenden Ungleichungen (2b), $(3 \mathrm{~b}),(18),(21),(25),(26)$ verlangen ja gewisse Eigenschaften lediglich von denjenigen $a_{\mu}^{(\nu)}$, bei denen beide Indices gewisse Grenzen überschreiten. Um im übrigen die Beziehungen zwischen den Limites der Zeilen (Colonnen) und denjenigen der Doppelfolge des genaueren festzustellen (s. § 4), schicken wir zunächst die folgenden Betrachtungen voraus. Dabei gilt alles, was beziiglich der Zeilen gesagt wird, mutatis mutandis auch für die Colonnen.

Die Terme $a_{\mu}^{(v)}$ jeder einzelnen Zeile (also $\nu$ beliebig, aber constant; $\mu=0,1,2, \cdots)$ besitzen stets einen unteren und einen oberen Limes:

$$
\left\{\begin{array}{ll}
\varliminf_{\mu=\infty} a_{\mu}^{(\nu)}=a^{(v)} & \text { bezw. }= \pm \infty, \\
\varlimsup_{\mu=\infty} a_{\mu}^{(\nu)}=\bar{a}^{(v)} & \text { bezw. }= \pm \infty,
\end{array} \quad(\nu=0,1,2, \cdots)\right.
$$

welche beiden eventuell auch zusammenfallen können. Im letzteren Falle "existirt" $\lim _{\mu=\infty} a_{\mu}^{(\nu)}$, in Zeichen*):

$$
\lim _{\mu=\infty} a_{\mu}^{(\nu)}=\varlimsup_{\lim } a_{\mu}^{(\nu)}=a^{(\nu)} \quad \text { bezw. }= \pm \infty,
$$

und die betreffende Zeile ist convergent bezw. eigentlich divergent.

Bildet man sodann die beiden Folgen:

$$
\left\{\begin{array}{l}
\varliminf_{\mu=\infty} a_{\mu}^{(0)}, \varliminf_{\mu=\infty} a_{\mu}^{(1)}, \cdots \varliminf_{\mu=\infty} a_{\mu}^{(\nu)}, \cdots \\
\varlimsup_{\mu=\infty} a_{\mu}^{(0)}, \varlimsup_{\mu=\infty} a_{\mu}^{(1)}, \ldots \varlimsup_{\mu=\infty} a_{\mu}^{(\nu)}, \ldots
\end{array}\right.
$$

(in denen eventưell auch beliebig viele Terme $+\infty,-\infty$ vorkommen können), so gehört zu jeder derselben wiederum ein unterer und ein oberer Limes, in Zeichen:

$$
\underline{\lim }_{\nu=\infty} \varliminf_{\mu=\infty} a_{\mu}^{(\nu)}, \cdots \varlimsup_{\nu=\infty} \varliminf_{\mu=\infty} a_{\mu}^{(\nu)}, \varliminf_{\nu=\infty} \varlimsup_{\mu=\infty} a_{\mu}^{(\nu)}, \varlimsup_{\nu=\infty} \varlimsup_{\mu=\infty} a_{\mu}^{(\nu)} .
$$

Die beiden äusseren dieser vier (eventuell auch sämmtlich oder theilweiø日 unter einander gleichen) Zahlen will ich als den iterirten unteren bezw. oberen Zeilen-Limes bezeichnen. Ans der unmittelbar : einleuchtenden $\mathrm{Be}-$ ziehung:

$$
\varliminf_{\mu=\infty} a_{\mu}^{(\nu)} \leqq \varlimsup_{\mu=\infty} a_{\mu}^{(\nu)} \quad(\nu=0,1,2, \cdots)
$$

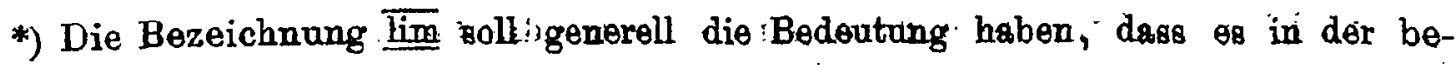
treffenden Formel freisteht, Џim oder 
folgt sodann:

$$
\left\{\begin{array}{l}
\varliminf_{\nu=\infty} \varliminf_{\mu=\infty} a_{\mu}^{(\nu)} \leqq \varlimsup_{\nu=\infty} \varlimsup_{\mu=\infty} a_{\mu}^{(\nu)} \leqq \varlimsup_{\nu=\infty} \varlimsup_{\mu=\infty} a_{\mu}^{(\nu)}, \\
\varliminf_{\nu=\infty} \varlimsup_{\mu=\infty} a_{\mu}^{(\nu)} \leqq \varlimsup_{\nu=\infty} \varlimsup_{\mu=\infty} a_{\mu}^{(\nu)} \leqq \varlimsup_{\nu=\infty} \varlimsup_{\mu=\infty} a_{\mu}^{(\nu)} * .
\end{array}\right.
$$

Sind also jene beiden äusseren Limites einander gleich, so fallen auch die beiden mittleren mit ihnen zusammen, d. h. in diesem Falle existirt ein eindeutig bestimmter

$$
\lim _{\nu=\infty} \varlimsup_{\mu=\infty} a_{\mu}^{(\nu)} \quad \text { (als endlich bezw. }+\infty \text { oder }-\infty \text { ), }
$$

der dann als iterirter Zeilen-Limes schlechthin bezeichnet werden möge.

2. Es verdient hervorgehoben $\mathrm{zu}$ werden, dass man den iterirten unteren bezw. oberen Zeilen-Limes allemal auch durch einfache Limites und zwar durch Grenzwerthe ächter Theilfolgen orsetzen kann**). Es gilt nämlich der Satz:

Man kann aus der Doppelfolge $\left(a_{\mu}^{(\nu)}\right)$ stets ächte Theilfolgen $\left(a_{m_{y}}^{\left(n_{v}\right)}\right),\left(a_{p_{y}}^{\left(q_{v}\right)}\right)$ so herausheben, dass:

$$
\lim _{\nu=\infty} a_{m_{\nu}}^{\left(n_{\nu}\right)}=\varliminf_{\nu=\infty} \varliminf_{\mu=\infty} a_{\mu}^{(\nu)}, \quad \lim _{v=\infty} a_{p_{\nu}}^{\left(q_{\nu}\right)}=\varlimsup_{\nu=\infty} \varlimsup_{\mu=\infty} a_{\mu}^{(\nu)} \text {. }
$$

Beim Beweise dieses Satzes haben wir drei Fälle zu unterscheiden, je

7) Dagegen kann sehr wohl:

aupfallen. Boi spiel:

$$
\varlimsup_{v=\infty} \varliminf_{\mu=\infty} a_{\mu}^{(\nu)}>\varliminf_{\nu=\infty} \varlimsup_{\mu=\infty} a_{\mu}^{(\nu)}
$$

also :

$$
a_{\mu}^{(v)}=(-1)^{\mu}+2\left(1+(-1)^{v}\right),
$$

und daher:

$$
\begin{aligned}
& a_{\mu}^{(2 v)}=(-1)^{\mu}+4, \\
& a_{\mu}^{(\Omega \nu+1)}=(-1)^{\mu},
\end{aligned}
$$

Dareus folgt weiter:

$$
\begin{aligned}
& \varliminf_{\mu=\infty} a_{\mu}^{(2 v)}=3, \quad \varlimsup_{\mu=\infty} a_{\mu}^{(8 \nu)}=5, \\
& \varliminf_{\mu=\infty} a_{\mu}^{(8 \nu+1)}=-1, \quad \varlimsup_{\mu=\infty} a_{\mu}^{(8 \nu+1)}=1 .
\end{aligned}
$$

d. b. in der That:

$$
\begin{aligned}
& \varlimsup_{v=\infty} \varliminf_{\mu=\infty} a_{\mu}^{(v)}=3, \\
& \varliminf_{v=\infty} \varlimsup_{\mu=\infty} a_{\mu}^{(v)}=1,
\end{aligned}
$$

$$
\varlimsup_{\nu=\infty} \varliminf_{\mu=\infty} a_{\mu}^{(\nu)}>\varliminf_{\nu=\infty} \varlimsup_{\mu=\infty} a_{\mu}^{(\nu)} .
$$

* Anders duisgedrücliti: Man kann jeden successiven Grenzübergang auch durth einen speciellen simultanen exsetzen. 
nachdem die in Frage kommenden Limites endlich oder unendlich mit noch näher zu specificirendem Vorzeichen ausfallen.

Fall I. Es sei zunächst:

$$
\varliminf_{\nu=\infty} \varliminf_{\mu=\infty} a_{\mu}^{(\nu)}=\underline{a} \quad \text { (wo } \underline{a} \text { eine bestimmte Zahl). }
$$

Aus der Folge:

$$
\varliminf_{\mu=\infty} a_{\mu}^{(0)}, \varliminf_{\mu=\infty} a_{\mu}^{(1)}, \ldots \varliminf_{\mu=\infty} a_{\mu}^{(\nu)}, \ldots
$$

muss sich dann eine unbegrenzte Folge endlicher Terme*)

$$
\varliminf_{\mu=\infty} a_{\mu}^{\left(n_{v}\right)}=\underline{a}^{\left(n_{v}\right)} \quad(\nu=0,1,2 \cdots)
$$

mit dem Grenzwerthe a herausheben lassen, also:

$$
\lim _{\nu=\infty} \underline{a}^{\left(n_{\nu}\right)}=a \quad \text { d. h. }=\varliminf_{\nu=\infty} \varliminf_{\mu=\infty} a_{\mu}^{(v)} \text {. }
$$

Da $\underline{a}^{\left(n_{v}\right)}$ den unteren Limes der Terme $a_{\mu}^{\left(n_{v}\right)}(\mu=0,1,2, \cdots)$ d. h. der $\left(n_{\nu}+1\right)^{\text {ten }}$ Zeile vorstellt, so muss es zu jedem $\varepsilon>0$ und zu jedem einzelnen $n_{v}$ unenalich viele Terme $a_{\mu_{\nu}}^{\left(n_{v}\right)}$ geben, sodass:

$$
\underline{a}^{(n v)}-\varepsilon \leqq a_{\mu_{v}}^{\left(n_{v}\right)} \leqq \underline{a}^{\left(n_{v}\right)}+\varepsilon \quad(\nu=0,1,2, \cdots) .
$$

Nimmt man also eine Folge positiver $\varepsilon_{\nu}$ mit den Grenzwerthe $\lim _{\nu=\infty} \varepsilon_{\nu}=0$, so existirt zunächst eine erste Zahl $m_{0}$, sodass:

$$
\underline{a}^{\left(n_{0}\right)}-\varepsilon_{0} \leqq \alpha_{m_{0}}^{\left(n_{0}\right)} \leqq \underline{a}^{\left(n_{0}\right)}+\varepsilon_{0} ;
$$

sodann eine erste Zahl $m_{1}$, welche gleichzeitig den beiden Bedingungen genügt:

$$
m_{1}>m_{0} \text { und: } \underline{a}^{\left(n_{1}\right)}-\varepsilon_{1} \leqq a_{m_{1}}^{\left(n_{1}\right)} \leqq \underline{a}^{\left(n_{1}\right)}+\varepsilon_{1} .
$$

So fortfahrend erhält man eine ächte Theilfolge $\left(a_{m_{v}}^{\left(n_{v}\right)}\right)$ von der Beschaffenheit, dass:

$$
\underline{a}^{\left(n_{\nu}\right)}-\varepsilon_{v} \leqq a_{m_{v}}^{\left(n_{v}\right)} \leqq \underline{a}^{\left(n_{v}\right)}+\varepsilon_{v} \quad(\nu=0,1,2, \cdots),
$$

und man findet somit, da $\lim _{\nu=\infty} \underline{a}^{\left(n_{\nu}\right)}$ nach Gl. (41) existirt:

$$
\lim _{\nu=\infty} a_{m_{\nu}}^{\left(n_{\nu}\right)}=\lim _{\nu=\infty} a^{\left(n_{\nu}\right)} \quad \mathrm{d} \cdot \mathrm{h}=\varliminf_{\nu=\infty} \lim _{\mu=\infty} a_{\mu}^{(v)}
$$

*) Die Voraussetzung (39) schliesst keineswegs aus, dass unter den Termen

$$
\varliminf_{\mu=\infty} a_{\mu}^{(v)} \quad(v=0,1,2, \cdots)
$$

der Werth $-\infty$ in endlicher Anzghl and der Werth $+\infty$ sogar unendlich oft vorkommt. 
Anslog ergiebt sich:

(45) $\lim _{v=\infty} a_{p_{v}}^{\left(q_{\nu}\right)}=\varlimsup_{\nu=\infty} \varlimsup_{\mu=\infty} a_{\mu}^{(v)}$, wenn: $\varlimsup_{\nu=\infty} \varlimsup_{\mu=\infty} a_{\mu}^{(\nu)}=\bar{a}$ (d. h. endlich), am einfachsten, wenn man beachtet, dass $\left(-\varlimsup_{\nu=\infty} \varlimsup_{\mu=\infty} a_{\mu}^{(\nu)}\right)$ den entsprechenden unteren Limes für die aus den Termen $\left(-a_{\mu}^{(\nu)}\right)$ gebildete Doppelfolge rorstellt.

Fall II. Es sei jetzt:

$$
\varliminf_{v=\infty} \varliminf_{\mu=\infty} a_{\mu}^{(\nu)}=-\infty
$$

Nimmt man eine Folge positiver Zahlen $G_{v}$ mit dem Grenzwerthe $\varliminf_{v=\infty} G_{v}=+\infty$, so muss sich auf Grund der Vorsussetzung (46) sus der Folge (40) eine unbegrenzte Folge von Termen $\varliminf_{\mu=\infty} a_{\mu}^{\left(x_{v}\right)}$ so herausheben lassen*), dass:

$$
\varliminf_{\mu=\infty} a_{\mu}^{\left(n_{\nu}\right)}<-G_{\nu} \quad(\nu=0,1,2, \cdots) .
$$

Aus der Bedeutung von $\underline{\lim }_{\mu=\infty} a_{\mu}^{\left(n_{v}\right)}$ folgt dann weiter, dass zu jedem einzelnen $n_{v}$ unenalich viele Terme $a_{\mu_{v}}^{\left(n_{v}\right)}$ existiren müssen, sodass:

$$
a_{\mu_{v}}^{\left(n_{v}\right)}<-G_{v} \quad(\nu=0,1,2, \cdots)
$$

Man kann daher ganz analog wie im Falle I zu $n_{0}, n_{1}, n_{2} ; \ldots$ eine steigende Folge natürlicher Zahlen $m_{0}, m_{1}, m_{2}, \cdots$ so auswählen, dass:

$$
a_{m_{\nu}}^{\left(n_{\nu}\right)}<-G_{\nu} \quad\left(m_{\nu}>m_{\nu-1}, n_{\nu}>n_{\nu-1}\right)
$$

und somit schliesslich:

$$
\varliminf_{\nu=\infty} a_{m_{v}}^{\left(n_{\nu}\right)}=-\infty \text { d. h. } \varliminf_{v=\infty} \varliminf_{\mu=\infty} a_{\mu}^{(v)} .
$$

Entsprechend ergiebt sich:

$$
\lim _{\nu=\infty} a_{p_{\nu}}^{\left(q_{v}\right)}=+\infty, \text { wenn: } \varlimsup_{v=\infty} \varlimsup_{\mu=\infty} a_{\mu}^{(v)}=+\infty .
$$

Fall III. Ist:

$$
\varliminf_{\nu=\infty} \varliminf_{\mu=\infty} a_{\mu}^{(\nu)}=+\infty \text { bezw. } \varlimsup_{\nu=\infty} \varlimsup_{\mu=\infty} a_{\mu}^{(\nu)}=-\infty,
$$

so folgt aus (37), dass auch:

$$
\varlimsup_{\nu \rightarrow \infty} \varlimsup_{\mu=\infty} a_{\mu}^{(\nu)}=+\infty \text { bezw. } \varliminf_{\nu=\infty} \varliminf_{\mu=\infty} a_{\mu}^{(v)}=-\infty \text {. }
$$

*) Diese Terme $\lim _{\mu=\infty} \alpha_{\mu}^{\left(n_{2}\right)}$ kömnen dabei für jedes einzelne v. endlich oder aneh olammtlich bezw. theilweise $=-\infty$ sein. 
Damit ist aber dieser Fall ohne weiteres auf Fall II zurückgeführt und der oben ausgesprochene Satz jetzt vollständig bewiesen.

3. Es erscheint für das folgende zweckmässig, in Bezug auf die successive Annäherung der Zahlen $a_{\mu}^{(v)}$ an die betreffenden Zeilen-Limites $\varlimsup_{\mu=\infty} a_{\mu}^{(\nu)}$ gewisse Unterscheidungen zu treffen und die verschiedenen Möglichkeiten durch geeignete Bezeichnungen zu charakterisiren.

Fasst man zunächst den einfachsten Fall in's Auge, dass jede Zeile convergirt, also etwa:

$$
\lim _{\mu=\infty} a_{\mu}^{(\nu)}=a^{(v)} \quad(v=0,1,2, \cdots),
$$

so besagt diese Voraussetzung lediglich folgendes: zu jedem (beliebig kleinen) $\varepsilon>0$ und für jedes einzelne $\nu$ existiren Zahlen $m_{\nu}$ und somit speciell eine kleinste Zahl $m_{v}{ }^{\prime}$, sodass:

$$
\left|a_{\mu}^{(\nu)}-a^{(\nu)}\right| \leqq \varepsilon, \quad \text { wenn: } \quad \mu \geqq m_{\nu}^{\prime} \quad(\nu=0,1,2, \cdots) .
$$

Dabei werden diese Zahlen $m_{\nu}^{\prime}$ (ausser mit $\varepsilon$ ) im allgemeinen mit $\nu$ veränderlich sein, und es erscheint insbesondere nicht ausgeschlossen, dass sie (sämmtlich oder theilweise) zugleich mit $\nu$ über alle Grenzen wachsen. Ebenso können auch die $\left|a^{(\nu)}\right|$ zugleich mit $\nu$ sämmtlich oder theilweise in's Unendliche wachsen. Wir sagen in jedem dieser beiden Fälle, die Zeilen seien ungleichmässig convergent.

Existirt dagegen für die Zahlen $m_{v}^{\prime}$ ein bestimmtes endliches Maximum $m$ und bleiben die $\left|a^{(v)}\right|$ unter einer endlichen Schranke $g$, so kann man die Bedingung (55) durch die folgende ersetzen:

$$
\left|a_{\mu}^{(\nu)}-a^{(\nu)}\right| \leqq \varepsilon, \quad \text { wenn: } \mu \geqq m \quad(\nu=0,1,2, \cdots),
$$

wobei jetzt $m$ eine (nur noch von $\varepsilon$ abhängende) für alle $\nu$ unveränderliche Zahl bedeutet und $\left|a^{(v)}\right|<g$ bleibt. In diesem Falle heissen die Zeilen gleichmässig convergent.

Sind alle Zeilen eigentlich divergent, also:

$$
\lim _{\mu=\infty} a_{\mu}^{(v)}=+\infty \text { bezw. }=-\infty \quad(\nu=0,1,2, \cdots),
$$

so besagt dies wiederum nur folgendes: zu jedem (beliebig grossen) $G>0$ und für jedes einzelne $\nu$ existiren Zahlen $m_{v}$ und speciell eine kleinste Zahl $m_{\nu}^{\prime}$, sodass:

(58) $a_{\mu}^{(v)}>G$ bezw. $<-G$, wenn $\mu \geq m_{\nu}^{\prime} \quad(y \doteq 0,1,2, \cdots)$.

Haben alsdann die $m_{\nu}^{\prime}$ ein bestimmtes endliches Maximum $m$, sodass die Bedingung (58) durch die folgende ersetzt werden känn:

$$
a_{\mu}^{(\nu)}>G \text { bezw. }<-G, \text { wenn } \mu \geqq m \quad(\nu=0,1,2, \cdots)
$$


so mögen die Zeilen als gleichmässig divergent bezeichnet werden; dagegen als ungleichmässig divergent, wenn ein solches Maximum nicht vorhanden ist, sodass also die $m_{v}^{\prime}$ gleichzeitig mit $v$ sämmtlich oder theilweise in's Unendliche wachsen.

Beispiele. 1) Die Zeilen der Folge $\left[\frac{\mu+\nu}{\mu \nu}\right]_{1}^{1}$ sind gleichmässig convergent. Denn man hat:

also:

$$
a_{\mu}^{(v)}=\frac{1}{\mu}+\frac{1}{\nu}, \quad \lim _{\mu=\infty} a_{\mu}^{(v)}=\frac{1}{\nu},
$$

$$
a_{\mu}^{(v)}-\lim _{\mu=\infty} a_{\mu}^{(v)}=\frac{1}{\mu} .
$$

Wird also $\varepsilon>0$ beliebig klein vorgeschrieben, darauf $m$ so fixirt, dass $\frac{1}{m} \leqq \varepsilon$, so wird:

$\left|a_{\mu}^{(1)}-\lim _{\mu=\infty} a_{\mu}^{(\nu)}\right| \leqq \frac{1}{\mu} \leqq \varepsilon$ für: $\mu \geqq m$ und $\nu=1,2,3, \ldots$

2) Die Zeilen der Folge $[\mu+\nu]_{0}^{0}$ sind gleichmässig divergent. Denn man hat:

$$
a_{\mu}^{(y)}=\mu+\nu, \quad \lim _{\mu=\infty} a_{\mu}^{(\nu)}=+\infty, \quad a_{\mu}^{(\nu)} \geqq \mu \quad(\nu=0,1,2, \cdots) .
$$

Wird also $G>0$ beliebig gross rorgeschrieben, sodann $m>G$ angenommen, so hat man:

$$
a_{\mu}^{(v)} \geqq m>G \text { für } \mu \geqq m \text { und } \nu=0,1,2, \cdots
$$

3) Die Zeilen der Folge $\left[\frac{(\mu-\nu)^{2}}{1+(\mu-\nu)^{2}}\right]_{0}^{0}$ sind ungleichmässig convergent. Aus:

folgt nämlich:

$$
a_{\mu}^{(\nu)}=\frac{(\mu-v)^{2}}{1+(\mu-v)^{2}}, \quad \lim _{\mu=\infty} a_{\mu}^{(v)}=1
$$

$$
\left|a_{\mu}^{(\nu)}-\lim _{\mu=\infty} a_{\mu}^{(\nu)}\right|=1-\frac{(\mu-\nu)^{2}}{1+(\mu-\nu)^{2}}=\frac{1}{1+(\mu-\nu)^{2}} .
$$

Versteht man unter $n$ eine beliebig grosse natürliche Zahl und setzt $\nu=n$ d. h. betrachtet man eine Zeile von beliebig hohem Range, so wird:

$$
\left|a_{n}^{(n)}-\lim _{\mu=\infty} a_{\mu}^{(n)}\right|=1
$$

Es ist somit nicht möglich, die fragliche Differenz durch Fixirung einer endlichen Maximalgrenze $\mu=m$ für alle $\nu$ numerisch unter ein beliebig: kleines $\varepsilon>0$ herabzudrücken. Das Wesen dieser ungleichmässigen $C o$ k vergene tritt noch anschaulicher hervor, wenn man sich die Glieder der betreffenden Doppelfolge in Form des Schema's (1) angeschrieben donkt: 


$$
\begin{aligned}
& 0 \quad \frac{1^{2}}{1+1^{2}} \quad \frac{2^{2}}{1+2^{2}} \frac{3^{2}}{1+3^{2}} \cdots \frac{\mu^{2}}{1+\mu^{2}} \\
& \frac{1^{2}}{1+1^{2}} \quad 0 \quad \frac{1^{2}}{1+1^{2}} \quad \frac{2^{2}}{1+2^{2}} \cdots \frac{(\mu-1)^{2}}{1+(\mu-1)^{2}} \cdots \\
& \frac{2^{2}}{1+2^{2}} \quad \frac{1^{2}}{1+1^{2}} \quad 0 \quad \frac{1^{2}}{1+1^{2}} \cdots \frac{(\mu-2)^{2}}{1+(\mu-2)^{2}} \cdots \\
& \frac{3^{2}}{1+3^{2}} \frac{2^{2}}{1+2^{2}} \quad \frac{1^{2}}{1+1^{2}} \quad 0 \quad \cdots \frac{(\mu-3)^{2}}{1+(\mu-3)^{2}} \cdots
\end{aligned}
$$

Man erkennt, dass die Terme jeder einzelnen Zeile schliesslich beständig wachsend der Grenze 1 zustreben. Aber dieses Zunehmen der Glieder beginnt erst jedesmal rechts ron dem Diagonalgliede, welches in jeder Zeile den Werth 0 hat: die successive Annäherung der Glieder an den Grenzwerth 1 wird mit jeder Zeile um eine Stelle weiter hinausgeschoben.

4) Auch die Zeilen der Folge $\left[\frac{\mu \nu}{\mu^{2}+\nu^{2}}\right]_{1}^{1}$ sind ungleichmässig comvergent. Man hat hier:

also:

$$
a_{\mu}^{(\nu)}=\frac{\mu \nu}{\mu^{2}+\nu^{2}}=\frac{1}{\frac{\mu}{\nu}+\frac{\nu}{\mu}}, \quad \lim _{\mu=\infty} a_{\mu}^{(\nu)}=0 \quad(\nu=1,2,3, \cdots),
$$

$$
\left|a_{\mu}^{(\nu)}-\lim _{\mu=\infty} a_{\mu}^{(\nu)}\right|=\frac{\mu \nu}{\mu^{2}+\nu^{2}}
$$

und daher für jedes noch so grosse $n$ :

$$
\left|a_{n}^{(n)}-\lim _{\mu=\infty} a_{\mu}^{(n)}\right|=\frac{1}{2}
$$

Die Terme jeder einzelnen Zeile convergiren hier schliesslich beständig abrehmend gegen 0; aber das Abnehmen der Glieder beginnt wieder jedesmal erst rechts ron dem Diagonalgliede, welches durchweg den Werth $\frac{1}{2}$ besitzt. Bedeutet ferner $p$ eine beliebige natürliche Zahl, so hat man:

$$
a_{p i}^{(v)}=\frac{p}{1+p^{2}}
$$

d. h. das Glied $\frac{p}{1+p^{2}}$, welches für $\nu=1$, d. h. in der ersten Zeile an der $p^{\text {ten }}$ Stelle steht, steht in der $n^{\text {ton }}$ Zeile erst an der $p n^{\text {ten }}$ Stelle: es findet also mit jeder neuen Zeile zugleich auch eine Verlangsamung der Gliederabnahme statt.

5) Als ungteichmässig convergent haben ferner die Zeilen der Folge: $\left[\nu+\frac{1}{\mu}\right]_{1}^{1}$ zu gelten. Zwar hat man hier:

$$
a_{\mu}^{(i)}=v+\frac{1}{\mu}, \quad \lim _{\mu=\infty} a_{\mu}^{(\nu)}=\nu
$$


also:

$$
a_{\mu}^{(\nu)}-\lim _{\mu=\infty} a_{\mu}^{(\nu)}=\frac{1}{\mu}
$$

und daher genau, wie bei dem Beispiele 1):

$$
\left|a_{\mu}^{(v)}-\lim _{\mu=\infty} a_{\mu}^{(v)}\right| \leqq \frac{1}{m} \leqq \varepsilon
$$

für $\mu \geqq m \geqq \frac{1}{\varepsilon}$ und $\nu=1,2,3, \ldots$.

Dagegen ist hier die Bedingung nicht erfüllt, dass die $\left|a^{(v)}\right|$ nnter einer endlichen Schranke bleiben, denn man hat:

$$
\lim _{\nu=\infty} a^{(v)}=\lim _{\nu=\infty} v=+\infty \text {. }
$$

6) Die Zeilen der Folge $\left[(\mu-\nu)^{2}\right]_{0}^{0}$ sind ungleichmässig divergent. Man hat:

$$
a_{\mu}^{(\nu)}=(\mu-v)^{2}, \quad \lim _{\mu=\infty} a_{\mu}^{(\nu)}=+\infty, \quad(\nu=0,1,2, \cdots) .
$$

Wird $G>0$ beliebig vorgeschrieben, so lässt sich kein $m$ so fixiren, dass:

$$
a_{\mu}^{(v)}>G \text { für } \quad \nu=0,1,2, \ldots
$$

Denn man hat, wie gross auch $n$ angenommen werden möge:

$$
\left.a_{n}^{(n)}=0 *\right)
$$

4. Die im vorstehenden eingeführten Begriffe der gleichmässigen Zeilen-Convergenz und -Divergenz gestatten noch eine für das folgende nützliche Verallgemeinerung.

Es werde zunächst angenommen, dass die Limites der Zeilen, zum mindesten nach Ansschluss einer endlichen Anzahl, numerisch unter einer festen Schranke bleiben (eine Annahme, die offenbar diejenige der ZeilenConvergenz gegen endlich bleibende Limites als speciellen Fall enthält), etwa:

$$
\underline{\lim } a_{\mu}^{(\nu)}=\underline{a}^{(\nu)}, \quad \overline{\lim } a_{\mu}^{(\nu)}=\bar{a}^{(\nu)}, \quad \text { wo: }\left\{\begin{array}{l}
\left|\underline{a}^{(\nu)}\right| \\
\left|\bar{a}^{(\nu)}\right|
\end{array}\right\}<g \text { für: } \nu \geqq n .
$$

Alsdann existirt zu jedem $\varepsilon>0$ und für jedes einzelne $\nu \geq n$ eine kleinste Zaihl $m_{v}^{\prime}$, sodess:

$$
\underline{a}^{(v)}-\varepsilon \leqq a_{\mu}^{(v)} \leqq \bar{a}^{(v)}+\varepsilon \quad \text { für: } \mu \geqq m_{\nu}^{\prime}, \quad \nu \geqq n .
$$

* Da die angefübrten Beispiele, ausser Nx. 5, in Bezug auf die beiden Indicés $\mu, \nu$ völlig symmetrisch gebaut sind, so können sie auch dazu dienen, um die in Rede stehenden Convergenz- und Divergenz-Eigenschaften der Zeilen anọh für die Colonnen exemplificiren. An Stelle von Beispiel 5) hätte man dann lediglich: $\left[\mu+\frac{1}{v}\right]_{1}^{1}$ za substituiren. 
Haben dann wiederum die $m_{v}^{\prime}$ ein endliches Maximum $m$, sodass also die Bedingung (61) durch die folgende ersetzt werden kann:

$$
\left.\underline{a}^{(v)}-\varepsilon \leqq a_{\mu}^{(v)} \leqq \bar{a}^{(v)}+\varepsilon \quad \text { für: } \mu \geqq m, \quad \nu \geqq n\left(\begin{array}{c}
\left|\underline{a}^{(\nu)}\right| \\
\left|\bar{a}^{(v)}\right|
\end{array}\right\}<g\right),
$$

so sollen die Zeilen für $v \geqq n$ gleichmässig begrenzt heissen; dagegen ungleichmässig begrenzt, wenn eine solche Zahl $m$ nicht existirt oder wenn die $\left|\underline{a}^{(v)}\right|$ und $\left|\bar{a}^{(v)}\right|$ nicht unter einer endlichen Schranke bleiben. Da die Bedingung (62) im Falle $\underline{a}^{(\nu)}=\bar{a}^{(\nu)}$ mit derjenigen für die gleichmässige Convergens der Zeilen bei $\nu \geqq n$ zusammenfällt (s. Ungl. (56)), so erscheint also diese letztere als specieller Fall der gleichmässigen Begrenzung.

Bestehen die fraglichen Bedingungen nur für einen der beiden Limites, d. h. hat man nur:

$$
\left\{\begin{array}{rll}
\underline{a}^{(\nu)}-\varepsilon \leqq a_{\mu}^{(v)} & \text { für: } \mu \geqq m, \nu \geqq n & \left(\left|\underline{a}^{(v)}\right|<g\right) \\
\text { oder: } \bar{a}^{(\nu)}+\varepsilon \geqq a_{\mu}^{(v)} & \text { für: } \mu \geqq m, \nu \geqq n & \left(\left|\bar{a}^{(\nu)}\right|<g\right),
\end{array}\right.
$$

so soll die Begrenzung der Zeilen eine einseitig gleichmässige heissen.

Bleiben die $\left|\underline{a}^{(v)}\right|,\left|\bar{a}^{(v)}\right|$ für $v \geq n$ nicht unter einer endlichen Schranke (woraus das ansloge für die $\left|a_{\mu}^{(\nu)}\right|$ bei gleichzeitig unbegrenzt wachsendem $\mu, \nu$ folgt), so soll speciell der Fall $\lim _{\nu=\infty} \varlimsup_{\mu=\infty} a_{\mu}^{(\nu)}=+\infty$ bezw. $-\infty$ betrachtet werden. Auf Grund dieser Voraussetzung existirt zunächst zu jedem (beliebig grossen) $G>0$ eine kleinste natürliche Zahl $n$, sodass: *)

(64) $\left\{\right.$ bezw. $\varlimsup_{\mu=\infty} a_{\mu}^{(\nu)}>-G, \quad$ also auch: $\varliminf_{\mu=\infty} a_{\mu}^{(\nu)}<-G$ für: $\nu \geqq n$.

Darsus folgt wiederum nur soviel, dass für jedes einzelne $v \geqq n$ eine kleinste Zahl $m_{\nu}^{\prime}$ existirt, sodass:

$$
a_{\mu}^{(v)}>G \text { bezw. }<-G \text { für: } \mu \geqq m_{\nu}^{\prime}, \nu \geqq n .
$$

Ist atadanin fứr die $m_{\nu}^{\prime}$ ein endliches Maximum $m$ vorhanden, sodass also:

$$
a_{\mu}^{(v)}>G \text { bezw. }<-G \text { für: } \mu \geqq m, \nu \geqq n,
$$

so sollen die Zeilen der Doppelfolge als gleichmässig anbegrenzt**) be-

- Dábei wird is im allgemeinen mit $G$ veränderlich oein (nămlich mit wachsenden Werthen ron $G$ gleichfalls zunehmen). Dies wăre nor dann nicht der Fall, rienn von oiner bestimmten Zeile ab, etwa für $\nu \geqq n^{\prime}$, durchweg:

$$
\varliminf_{\mu}^{(v)}={ }_{\lim } a_{\mu}^{\nu}=+\infty \text { bezw. }=-\infty \text {. }
$$

Dimin ganugt nitientich die Annahme $n=n^{\prime}$ bei jedem beliebigen $G$.

- Dai Ausdruck bezieht sich wesentlich auf die Gesammtheit der Zeilen. Jede einzelns Zeile kann debei immerhin begrenzt (d. h. mit endlichen Limites versehen),
sogar convergent sein (s. Beispiel 9)). 
zeichnet werden. Die Vergleichung der Bedingung (66) mit der für gleichmässige Divergenz geltenden Bedingung (58) zeigt wiederum, dass diese letztere Eigenschaft einen speciellen Fall der soeben definirten darstellt.

Beispiele. 7) Die Zeilen der Folge $\left[(-1)^{\mu+\nu} \frac{\mu+\nu}{\mu \nu}\right]_{1}^{1}$ sind gleichmässig begrenzt. Man hat:

$$
\begin{array}{ll}
a_{\mu}^{(v)}=(-1)^{\mu+\nu}\left(\frac{1}{\mu}+\frac{1}{\nu}\right), \text { also: } & \underline{a}^{(v)}=\varliminf_{\mu=\infty} a_{\mu}^{(v)}=-\frac{1}{\nu}, \\
\bar{a}^{(v)} & =\varlimsup_{\mu=\infty} a_{\mu}^{(v)}=+\frac{1}{\nu} .
\end{array}
$$

Da nun:

so folgt:

$$
-\frac{1}{\mu}-\frac{1}{\nu} \leqq a_{\mu}^{(\nu)} \leqq \frac{1}{\mu}+\frac{1}{\nu} \quad(\nu=1,2,3, \cdots),
$$

und daher:

$$
\underline{a}_{\nu}-\frac{1}{\mu} \leqq a_{\mu}^{(\nu)} \leqq \bar{a}_{\nu}+\frac{1}{\mu} \quad(\nu=1,2,3, \cdots)
$$

$\underline{a}_{\nu}-\varepsilon \leqq a_{\mu}^{(\nu)} \leqq \bar{a}_{\nu}+\varepsilon \quad$ für: $\mu \geqq m \geqq \frac{1}{\varepsilon}, \quad \nu=1,2,3, \ldots$

8) Die Zeilen der Folge $\left[(-1)^{\mu+v} \frac{2+(\mu-v)^{2}}{1+(\mu-\nu)^{2}}\right]_{0}^{0}$ sind ungleichmässig begrenzt. Man bat:

$$
\begin{aligned}
a_{\mu}^{(\nu)}=(-1)^{\mu+v} \frac{2+(\mu-v)^{2}}{1+(\mu-v)^{2}}, \quad \text { also: } & \underline{a}^{(v)}=\varliminf_{\mu=\infty} a_{\mu}^{(v)}=-1, \\
& \bar{a}^{(v)}=\varlimsup_{\mu=\infty} a_{\mu}^{(v)}=+1 .
\end{aligned}
$$

Andererseits wird, für jedes noch so grosse $n$ :

$$
a_{n}^{(n)}=2, \quad a_{n \pm 1}^{(n)}=-\frac{8}{2},
$$

d. h. für jede Zeile von beliebig hohem Range $n$ liegen das $n \pm 1^{\text {to }}$ und $n^{\text {to }}$ Glied ausserhalb des Intervalles $\left(\underline{a}^{(n)}-\varepsilon, \bar{a}^{(n)}+\varepsilon\right)=(1-\varepsilon, 1+\varepsilon)$.

9) Die Zeilen der Folge $\left[\nu+\frac{1}{\mu}\right]_{1}^{1}$, welche oben (Beispiel 5)) als ungleichmässig convergent erkannt wurden, sind andererseits gleichmässig unbegrenat. Denn man hat

$$
a_{\mu}^{(\nu)}=\nu+\frac{1}{\mu}>\nu .
$$

Wird also $n \geqq G$ angenommen; so folgt:

$$
a_{\mu}^{(v)} \geqq n>G \text { für: } \nu \geqq n, \mu \geqq 1 .
$$

10) Gleichmässig unbegrenzt sind such die Zeilen der Folge $\left[\nu+\left(1+(-1)^{\mu}\right) \mu\right]_{0}^{0}$. Man hat hier:

$$
\begin{array}{ll}
a_{\mu}^{(v)}=v, & \text { wenn } \mu \text { gerade, } \\
a_{\mu}^{(v)}=v+2 \mu, & \text { wenn } \mu \text { ungerade }
\end{array}
$$


und daher:

$$
\varliminf_{\mu=\infty} a_{\mu}^{(\nu)}=\nu, \quad \varlimsup_{\mu=\infty} a_{\mu}^{(\nu)}=+\infty
$$

im übrigen für $n>G$ :

$$
a_{\mu}^{(v)} \geq n>G \quad \text { für: } \quad \nu \geqq n, \mu \geqq 0 .
$$

11) Setzt man:

so folgt:

$$
a_{\mu}^{(v)}=\lim _{p=\infty} \frac{\mu^{p-1}-\nu^{p-1}}{\mu^{p}+\nu^{p}} \cdot \mu \nu(\mu=1,2,3, \cdots ; \nu=1,2,3, \cdots),
$$

$$
a_{\mu}^{(\nu)}\left\{\begin{array}{l}
=\lim _{p=\infty} \frac{1-\left(\frac{\nu}{\mu}\right)^{p-1}}{1+\left(\frac{\nu}{\mu}\right)^{p}} \cdot \nu=\nu, \quad \text { wenn: } \mu>\nu, \\
=\lim _{p=\infty} \frac{\left(\frac{\mu}{\nu}\right)^{p-1}-1}{\left(\frac{\mu}{\nu}\right)^{p}+1} \cdot \mu=-\mu, \text { wenn: } \mu<\nu . \\
. \quad \cdot . \cdot . \cdot=0, \quad \text { wenn: } \mu=\nu .
\end{array}\right.
$$

Folglich wird:

$$
\varliminf_{\mu=\infty} a_{\mu}^{(\nu)}=\varlimsup_{\mu=\infty} a_{\mu}^{(\nu)}=\nu,
$$

d. h. die Zeilen sind convergent, aber ungleichmässig, weil der Grenzwerth $\nu$ schliesslich in's Unendliche wächst, übrigens aber auch darum, weil (für jedes noch so grosse $n$ ) $a_{n}^{(n)}=0, a_{n-\lambda}^{(n)}=-(n-\lambda)$. Aus diesem letzteren Grunde sind dann aber die Zeilen auch nicht einmal gleichmässig unbegrenzt.*)

$\S 4$.

Beziehuingen zwischen den iterirten Limites der Zeilen (Colonnen) und den Limites der Doppelfolge.

1. Mit Htulfè der im vorigen Paragraphen eingeführten Unterscheidungen lassen sich dio Beziehungen zwischen den iterirten (Zeilen- bezw. Colọnnen-) Limites und den in $\$ \S 1,2$ betrachteten Doppel-Limites in sehr allgemeiner Weise feststellen. Zunächst ergiebt sich der folgende Satz:

(I) Der iterite untere 'bezw. obere Limes ist niemals kleiner besw. gitösser, als der entsprechende Doppel-Limes, d. h. man hat stets:

*) Die Beispielo 7), 8)!, 11) gelten wiederum anch unmittelbar für die entsprechenden Erscheinurngen bezüglich der Colonnen; die Beispiele 9), 10) nsch Vertauschung von $\mu$ und $\nu$. 


$$
\varliminf_{\mu, \nu=\infty} a_{\mu}^{(\nu)}\left\{\begin{array}{l}
\leqq \lim _{\nu=\infty} \prod_{\mu=\infty} a_{\mu}^{(v)} \leqq \varlimsup_{v=\infty} \varlimsup_{\mu=\infty} a_{\mu}^{(\nu)} \\
\leqq \lim _{\mu=\infty} \varliminf_{\nu=\infty} a_{\mu}^{(\nu)} \leqq \varlimsup_{\mu=\infty} \varlimsup_{\nu=\infty} a_{\mu}^{(\nu)}
\end{array}\right\} \leqq \varlimsup_{\mu, \nu=\infty} a_{\mu}^{(\nu)} .
$$

Beweis. Nach dem Satze in Nr. 2 des vorigen Paragraphen kann man jeden der betreffenden iterirten Limites durch den Limes einer ächten Theilfolge ersetzen. Nach § 2, Nr. 5 existirt aber keine ächte Theilfolge, deren Grenzwerth $<\lim _{\mu, \nu=\infty} a_{\mu}^{(\nu)}$ oder $>\varlimsup_{\mu, \nu=\infty} a_{\mu}^{(\nu)}$ : somit ist der fragliche Satz bewiesen. -

Fasst man insbesondere den Fall in's Auge, dass ${\underset{\mu i m}{\mu, v=\infty}}_{\mu} a_{\mu}^{(v)}=\varlimsup_{\mu, v=\infty} a_{\mu}^{(v)}$, so resultirt aus (I) unmittelbar der folgende Specialsatz*):

(Ia) Man hat stets:

$$
\left.\begin{array}{l}
\lim _{v=\infty} \varlimsup_{\mu=\infty} a_{\mu}^{(v)} \\
\lim _{\mu=\infty} \varlimsup_{\nu=\infty} a_{\mu}^{(\nu)}
\end{array}\right\}=\lim _{\mu, v=\infty} a_{\mu}^{(\nu)},
$$

sobald der letztere Grenzwerth existirt.

Ist die Doppelfolge $\left(a_{*}^{(v)}\right)$ monoton, so gilt das gleiche von den Zeilen- und Colonnen-Folgen. In diesem Falle existiven also auch $\lim _{\mu=\infty} a_{\mu}^{(\nu)}$, $\lim _{v=\infty} a_{\mu}^{(v)}$, sodass für eine monotone Doppelfolge unter allen Umständen die Beziehung besteht:

$$
\lim _{\nu=\infty} \lim _{\mu=\infty} a_{\mu}^{(\nu)}=\lim _{\mu=\infty} \lim _{\nu=\infty} a_{\mu}^{(\nu)}=\lim _{\mu, \nu=\infty} a_{\mu}^{(\nu)}
$$

Aus (68) folgt des weiteren, dass die Existenz von $\lim _{\mu, v=\infty} a_{\mu}^{(\nu)}$ eine hinreichende Bedingung für die Beziehung:

$$
\lim _{\nu=\infty} \varlimsup_{\mu=\infty} a_{\mu}^{(\nu)}=\lim _{\mu=\infty} \varlimsup_{\nu=\infty} a_{\mu}^{(\nu)}
$$

und diese letztere somit umgekehrt eine nothwendige Bedingung für die Existenz von $\lim _{\mu, \nu \dot{S}_{-\infty}} a_{\mu}^{(v)}$ bildet. Daraus ergiebt sich insbesondere, dass $\lim _{\mu, v=\infty} a_{\mu}^{(\nu)}$ sicher nicht existirt, wenn die beiden Grenzwerthe ( 70$)$ verschieden augfallen. (Beispiele: $\left[\frac{\mu}{\mu+\nu}\right]_{1}^{1},\left[2^{-\nu}\right]_{1}^{1},\left[\left(\frac{\mu}{\mu+1}\right)^{\nu}\right]_{1}^{1}$.

Andererseits erscheint die Existenz von $\lim _{\mu, v=\infty} a_{\mu}^{(v)}$ keineswegs als nothwendig für das Bestehen der Beziehung (70) (s. \& 3, Beisp. 3), 4), 5), 6\% 10), 11)) y diese letztere also nicht als hinreichend für. die Existisns

*) Vgl. Münch. Sitz.-Ber. a. 8. O. p. 105; desgl. Bd. 28 (1898), p. . 63 ? 
$\lim _{\mu, \nu=\infty} a_{\mu}^{(v)}$. Neben den eben citirten Beispielen möchte ich als besonders lehrreich noch das folgende anführen:

$$
a_{\mu}^{(\nu)}=(-1)^{\mu} \cdot \frac{\mu^{8} \nu^{3}}{\mu^{8}+\nu^{6}} \quad(\mu=1,2,3, \cdots ; \nu=1,2,3, \cdots) .
$$

Man hat hier zunächst:

also auch:

$$
\lim _{\mu=\infty} a_{\mu}^{(v)}=0, \quad \lim _{\nu=\infty} a_{\mu}^{(\nu)}=0,
$$

$$
\lim _{\nu=\infty} \lim _{\mu=\infty} a_{\mu}^{(\nu)}=0, \quad \lim _{\mu=\infty} \lim _{\nu=\infty} a_{\mu}^{(\nu)}=0 .
$$

Ferner ergiebt sich:

$$
\lim _{v=\infty} a_{v}^{(\nu)}=\lim _{\nu=\infty}(-1)^{v} \cdot \frac{\nu^{2}}{1+v^{8}}=0,
$$

and sogar allgemein, für jedes ganzzahlige $p \geqq 1, q \geqq 0$ :

$$
\begin{aligned}
& \lim _{v=\infty} a_{p \nu+q}^{(v)}=\lim _{v=\infty}(-1)^{p v+q} \cdot \frac{(p \nu+q)^{q} \nu^{8}}{(p \nu+q)^{3}+\nu^{6}}=0, \\
& \lim _{\nu=\infty} a_{\nu}^{(p \nu+q)}=\lim _{\nu=\infty}(-1)^{\nu} \quad \cdot \frac{\nu^{2}(p \nu+q)^{2}}{\nu^{8}+\left(p^{\nu}+q\right)^{6}}=0 .
\end{aligned}
$$

Die Folge $\left(a_{\mu}^{(\nu)}\right)$ hat also die Eigenschaft, dass nicht nur die iterirten Limites und der Grenzwerth der Hauptdiagonale den Werth 0 haben, sondern dass alle in ihr enthaltenen "geradlinigen"*) Theilfolgen gegen 0 convergiren. Nichts destoweniger hat man:

âlso:

$$
a_{v^{2}}^{(\nu)}=(-1)^{v^{2}} \cdot \frac{1}{2} \nu
$$

$$
\lim _{\nu=\infty} a_{\nu^{2}}^{(\nu)}= \pm \infty, \text { jenachdem } v \text { gerade } \text { oder ungerade, }
$$

und daher:

$$
\lim _{\mu, i=\infty} a_{\mu}^{(v)}=-\infty, \quad \varlimsup_{\mu, v=\infty} a_{\mu}^{(i)}=+\infty .
$$

2. Ebenso wenig wie das Bestehen der Beziehung (70) die Existenz von $\lim _{\mu, v \pm \infty} a_{\mu}^{(j)}$ und somit (nach Satz Ia) das Zusammenfallen der iterirten Limites mit dem Doppel-Limes verbürgt, brauchen allgemein der iterirte untere und obere Limes mit dem unteren bezw. oberen Doppel-Limes zusammenzufallon. Man bemerke zunächst, dass ein solches Zusammenfallen auf Gruad der Beriehung (67) allemal dann stattfindet, wenn der iterirte untere Limes den Werth $-\infty$, der obere den Werth $+\infty$ hat. Im übrigen

*) Vgl. p. 300, Fassri.t. 
ergiebt sich für das Zusammenfallen der betreffenden Limites zunächst die folgende hinreichende Bedingung:

(II) Sind die Zeilen (Colonnen) der Doppelfolge nach eventuellem Ausschlusse einer endlichen Anzahl gleichmässig be-

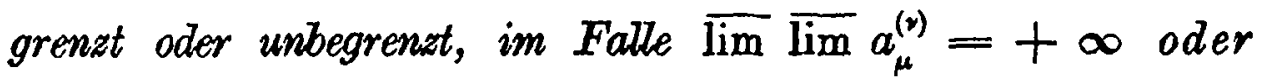
$\underline{\lim } \underline{\lim } a_{\mu}^{(\nu)}=-\infty$ wenigstens einseitig gleichmässig begrenzt, so ist:

$$
\left\{\begin{aligned}
\varliminf_{\nu=\infty} \lim _{\mu=\infty} a_{\mu}^{(v)}=\lim _{\mu, \nu=\infty} a_{\mu}^{(v)}, & \varlimsup_{\mu=\infty} \varlimsup_{\nu=\infty} a_{\mu}^{(v)}=\varlimsup_{\mu, \nu=\infty} a_{\mu}^{(v)}, \\
\text { (bezw. } \varliminf_{\mu=\infty} \varliminf_{\nu=\infty} a_{\mu}^{(v)}=\varliminf_{\mu, v=\infty} a_{\mu}^{(v)}, & \left.\varlimsup_{\mu=\infty} \varlimsup_{\nu=\infty} a_{\mu}^{(v)}=\varlimsup_{\mu, \nu=\infty} a_{\mu}^{(v)}\right) .
\end{aligned}\right.
$$

Beweis. Fall I. Sind die Zeilen zum mindesten für $v \geqq n^{\prime}$ gleichmässig begrenzt, und setzt man:

$$
\varliminf_{\mu=\infty} a_{\mu}^{(v)}=\underline{a}^{(v)}, \quad \varlimsup_{\mu=\infty} a_{\mu}^{(\nu)}=\bar{a}^{(\nu)},
$$

so liegen (s. \&3, Nr. 4) die $a^{(v)}$, $\vec{a}^{(v)}$ für $v \geqq n^{\prime}$ innerbalb endlicher Schranken und $z \mathfrak{u}$ jedem $\varepsilon>0$ lässt sich $m$ so fixiren, dass:

$$
\underline{a}^{(\nu)}-\frac{\varepsilon}{3} \leqq a_{\mu}^{(\nu)} \leqq \bar{a}^{(\nu)}+\frac{\varepsilon}{3} \text { für: } \mu \geqq m, \nu \geqq n^{\prime} \text {. }
$$

Wird sodann gesetzt:

$$
\varliminf_{v=\infty} \underline{a}^{(\nu)}=\underline{a}, \quad \varlimsup_{\nu=\infty} \bar{a}^{(\nu)}=\bar{a},
$$

so kann man ein $n \geqq n^{\prime}$ so annehmen, dess:

$$
\underline{a}-\frac{\varepsilon}{3} \leqq \underline{a}^{(v)} \leqq \bar{a}^{(v)} \leqq \bar{a}+\frac{\varepsilon}{3} \quad \text { für }: \quad \nu \geqq n,
$$

sodass sich durch Combination von (73) und (75) ergiebt:

$$
\underline{a}-\frac{2 \varepsilon}{3} \leqq a_{\mu}^{(\nu)} \leqq \bar{a}+\frac{2 \varepsilon}{3} \text { fur: } \mu \geqq m, \nu \geqq n .
$$

Da hiernach die $a_{\mu}^{(v)}$ für $\mu \geqq m, \nu \geqq n$ innerhalb endlicher Schranken bleiben, so hat man:

$$
\varliminf_{\mu, \nu=\infty} a_{\mu}^{(v)}=\underline{A}, \quad \varlimsup_{\mu, \nu=\infty} a_{\mu}^{(\nu)}=\bar{A},
$$

wo $\underline{A}, \bar{A}$ bestimmte Zahlen vorstellen. Wegen der Bedeutung dieser Zahlen $A, \bar{A}$ (s. $\S 2$, Ungl. 24) giebt es alsdann ächte Theilfolgen $\left(a_{m_{v}}^{n_{v}}\right),\left(a_{p_{v}}^{q_{v}}\right)$, sodass:

$$
a_{m_{v}}^{\left(n_{v}\right)} \leqq \underline{A}+\frac{\varepsilon}{3}, \quad \bar{A}-\frac{\varepsilon}{3} \leqq a_{p_{v}}^{\left(q_{v}\right)} .
$$


Da es hierbei freisteht, von vornherein $m_{v} \geqq m, n_{v} \geqq n$ anzunehmen und andererseits die Ungleichungen (76) bestehen bleiben, wenn man die beliebigen $\mu, \nu$ durch die specielleren $m_{\nu}, n_{\nu}$ bezw. $p_{\nu}, q_{\nu}$ ersetzt, so resultiren aus der Verbindung von (76) und (78) die folgenden Beziehungen:

$$
a-\frac{2 \varepsilon}{3} \leqq \underline{A}+\frac{\varepsilon}{3}, \quad \bar{A}-\frac{\varepsilon}{3} \leqq \bar{a}+\frac{2 \varepsilon}{3},
$$

anders geschrieben:

$$
\underline{a} \leqq \underline{A}+\varepsilon, \quad \bar{a} \geqq \bar{A}-\varepsilon,
$$

d. h., da $\varepsilon$ jede noch so kleine positive Zahl vorstellen kann:

$$
\underline{a} \leqq \underline{A}, \quad \bar{a} \geqq \bar{A}
$$

and, da die Möglichkeit $a<\underline{A}, \bar{a}>\bar{A}$ durch Satz I (Ungl. (67)) definitiv ausgeschlossen erscheint, schliesslich:

$$
\underline{a}=\underline{A}, \quad \bar{a}=\bar{A},
$$

d. h.

$$
\varliminf_{\nu=\infty} \varliminf_{\mu=\infty} a_{\mu}^{(\nu)}=\varliminf_{\mu, \nu=\infty} a_{\mu}^{(\nu)}, \quad \varlimsup_{\nu=\infty} \varlimsup_{\mu=\infty} a_{\mu}^{(\nu)}=\varlimsup_{\mu, \nu=\infty} a_{\mu}^{(\nu)} \cdot-
$$

Fall II. Es seien jetzt die Zeilen für $\nu \geqq n^{\prime}$ gleichmässig unbegrenzt, also (s. Ungl. (66)) etwa:

$$
a_{\mu}^{(v)}>G \text { für: } \mu \geqq m, \quad \nu \geqq n \geqq n^{\prime} .
$$

Daraus ergiebt sich zunächst, dass auch:

und daher:

$$
\varliminf_{\mu=\infty} a_{\mu}^{(\nu)}>G \quad \text { für: } \quad v \geqq n,
$$

(84) $\varliminf_{\nu=\infty} \varliminf_{\mu=\infty} a_{\mu}^{(v)}=+\infty$, also a fortiori auch: $\varlimsup_{\nu=\infty} \varlimsup_{\mu=\infty} a_{\mu}^{(\nu)}=+\infty$.

Sodann folgt aber aus Ungl. (67) oder auch unmittelbar aus der Vergleichang der Bedingung (83) mit derjenigen für die eigentliche Divergenz der Doppelfolge (s 1 , Ungl. (3a))*), dass auch:

sodass also schliesslich:

$$
\lim _{\mu, \nu=\infty} a_{\mu}^{(v)}=+\infty
$$

$$
\varlimsup_{\nu=\infty} \varlimsup_{\mu=\infty} a_{\mu}^{(v)}=\varlimsup_{\mu, \nu=\infty} a_{\mu}^{(v)}=+\infty
$$

*) Die analytische Formulirung dérjenigen Eigenschaft, wielchè hier als „gleichmässige Unbegrenztheit" der Zeilen bezeichnet wird, ist in Wärheit init der nothwendigen und hinreichenden Bedingung für :die. eigentliche Divergenz der Doppelfolge identisch. 
oder einfacher geschrieben:

$$
\lim _{\nu=\infty} \varlimsup_{\mu=\infty} a_{\mu}^{(\nu)}=\lim _{\mu, \nu=\infty} a_{\mu}^{(\nu)}=+\infty .
$$

Analog ergiebt sich im Falle:

dess :

$$
\text { . } a_{\mu}^{(\nu)}<-G \text { für: } \mu \geqq m, \nu \geqq n \geqq n^{\prime},
$$

$$
\lim _{\nu=\infty} \varlimsup_{\mu=\infty} a_{\mu}^{(\nu)}=\lim _{\mu, \nu=\infty} a_{\mu}^{(\nu)}=-\infty
$$

Fall III. Es sei:

$\varlimsup_{\nu=\infty} \varlimsup_{\mu=\infty} a_{\mu}^{(\nu)}=+\infty$, dagegen $\varliminf_{\nu=\infty} \lim _{\mu=\infty} \equiv \lim _{\nu=\infty} \underline{a}^{(\nu)}=\underline{a}$ (endlich) und die Zeilen für $v \geqq n^{\prime}$ in Bezug auf die $\underline{a}^{(v)}$ gleichmässig begrenzt, sodass also:

$$
\underline{a}^{(\nu)}-\frac{\varepsilon}{3}<a_{\mu}^{(v)} \text { für: } \mu \geq m, \quad \nu \geq n^{\prime} .
$$

Alsdann lässt sich wiederum ein $n \geq n^{\prime}$ so fixiren, dass:

$$
\underline{a}-\frac{\varepsilon}{3} \leqq \underline{a}^{(v)} \quad \text { für: } \quad \nu \geqq n,
$$

sodass also:

$$
\underline{a}-\frac{2 \varepsilon}{B} \leqq a_{\mu}^{(\nu)} \quad \text { für: } \mu \geq m, \nu \geqq n .
$$

Da sodann: $\varliminf_{\mu, \nu=\infty} a_{\mu}^{(v)} \leqq \varliminf_{v=\infty} \frac{\lim _{\mu=\infty}}{a_{\mu}^{(v)}}$ d. h. $\leqq \underline{a}$ und andererseits für alle $a_{\mu}^{(\nu)}$ bei $\mu \geqq m, \nu \geqq n$ nach Ungl. (89) $a_{\mu}^{(v)} \geqq \underline{a}-\frac{2 \varepsilon}{3}$, so ist:

$$
\lim _{\mu, \nu=\infty} a_{\mu}^{(v)}=\underline{A}
$$

eine bestimmte endliche Zahl.

Es existirt daher wiederum eine ächte Theilfolge $\left(a_{m_{y}}^{\left(n_{v}\right)}\right)$, sodass (s. § 2, Ungl. (24)):

$$
a_{m_{v}}^{\left(n_{v}\right)} \leqq \underline{A}+\frac{\varepsilon}{8}
$$

Daraus folgt aber in Verbindung mit Ungl. (8!)), dass:

$$
\underline{a}-\frac{2 \varepsilon}{3} \leqq \underline{A}+\frac{\varepsilon}{3} \text { d. h. } \underline{a} \leqq \underline{A}+\varepsilon
$$

und schliesslich, gensn wie im Falle I:

$$
\underline{a}=\underline{A} \quad \text { d. h. } \varliminf_{\nu=\infty} \varliminf_{\mu=\infty} a_{\mu}^{(\nu)}=\varliminf_{\mu, \nu=\infty} a_{\mu}^{(\nu)} .
$$


Ueberdies ergiebt sich aus der Voraussetzung: $\varlimsup_{\nu=\infty} \varlimsup_{\mu=\infty} a_{\mu}^{(\nu)}=+\infty$ auf Grund von Ungl. (67), dass auch:

$$
\varlimsup_{\mu, v=\infty} a_{\mu}^{(\nu)}=+\infty, \text { also: }=\varlimsup_{\nu=\infty} \varlimsup_{\mu=\infty} a_{\mu}^{(\nu)} \text {. }
$$

Das analoge gilt sodann im Falle: $\lim _{\nu=\infty} \lim _{\mu=\infty} a_{\mu}^{(\nu)}=-\infty, \varlimsup_{\nu=\infty} \varlimsup_{\mu=\infty} a_{\mu}^{(\nu)}=\bar{a}$.

Da sich die rorstehenden Betrachtungen ohne weiteres auch auf die Colonnen übertragen lassen, so erscheint hiermit der oben ausgesprochene Satz in allen seinen Theilen bewiesen.

3. Nimmt man im Falle I des vorigen Satzes $\varliminf_{\nu=\infty} \varliminf_{\mu=\infty} a_{\mu}^{(\nu)}$ und $\varlimsup_{\nu=\infty} \varlimsup_{\mu=\infty} a_{\mu}^{(\nu)}$ als zusammenfallend an, so gewinnt man unmittelbar den folgenden Specialsatz:

(II a) Sind die Zeilen der Doppelfolge $\left(a_{\mu}^{(v)}\right)$ nach eventuellem Ausschlusse einer endlichen Anzahl gleichmässig begrenzt und existirt:

$$
\left.\lim _{\nu=\infty} \varlimsup_{\mu=\infty} a_{\mu}^{(v)}=a^{*}\right),
$$

so convergirt die Doppelfolge und man hat:

$$
\lim _{\mu, v=\infty} a_{\mu}^{(v)}=a
$$

Zugleich gestattet der Fall (II) des obigen Satzes die folgende Formulirung:

(II b) Sind die Zeilen der Doppelfolge $\left(a_{\mu}^{(\nu)}\right)$ nach eventuellem Ausschlusse einer endlichen Anzahl gleichmässig unbegrenzt, so ist nicht nur:

$$
\lim _{\nu=\infty} \varlimsup_{\mu=\infty} a_{\mu}^{(v)}=+\infty \text { bezw. }=-\infty,
$$

sondern die Doppelfolge selbst isteigentlich divergent, sodass also:

$$
\lim _{\mu, \nu=\infty} a_{\mu}^{(\nu)}=+\infty \text { bezw. }=-\infty .
$$

Das analoge gilt wiederum bezüglich der Colonnen.

Die Sätzè (II a), (IIb) zeigen insbesondere, dass für die Convergenz bezw. eigentliche Divergenz der Dọppelfolge die Convergenz bezw. eigentliche

*) Dass in diesem Falle $\lim _{\nu=\infty} \varliminf_{\mu=\infty} a_{\mu}^{(\nu)}$ stets eine endliche Zahl $a$ ist, folgt unmittelbar aus der Voraussetzung der gleichmäassigen Begrenzung: denn diese erheischt, dass $\varliminf_{\mu=\infty} a_{\mu}^{(\nu)}, \varlimsup_{\mu=\infty} a_{\mu}^{(v)}$ zum mindesten für $\nu \geqq m$ zwischen zwei endlichen Schranken bleiben (s. \& 3, Nr. 4). 
Divergene keiner einzigen Zeile (Colonne) erforderlich ist. (Beispiel einer convergenten Doppelfolge mit durchweg oscillirenden Zeilen s. § 3, Nr. 4, Beisp. 7); eigentlich divergente Folge mit durchweg convergenten Zeilen: ebendas. Beisp. 9); eigentlich divergente Folge, deren Zeilen innerhalb endlicher Grenzen oscilliren: $\left[\left(2+(-1)^{\mu}\right) \nu\right]_{0}^{0}$; eigentlich divergente Folge, deren Zeilen einen exdlichen unteren und unendlichen oberen Limes besitzen: a. a. O. Beisp. 10)).

3. Ich will nun untersuchen, in wieweit die in Satz (II), (II a), (IIb) angegebenen, für das Zusammenfallen. der iterirten und der entsprechenden Doppel-Limites hinreichenden Bedingungen sich zugleich auch als nothwendige erweisen, d. h. in wieweit die betreffenden Sätze umkehrbar sind.

Dass dies für den allgemeinen Satz (II) nicht der Fall ist, sodass man also aus den Beziehungen:

$$
\varliminf_{\nu=\infty} \varliminf_{\mu=\infty} a_{\mu}^{(\nu)}=\varliminf_{\mu, \nu=\infty} a_{\mu}^{(\nu)}, \quad \varlimsup_{\nu=\infty} \varlimsup_{\mu} a_{\mu}^{(\nu)}=\varlimsup_{\mu, \nu=\infty} a_{\mu}^{(\nu)}
$$

nicht auf die gleichmässige Begrenztheit oder Unbegrenztheit der Zeilen schliessen darf, zeigt die folgende Ueberlegung. Es sei $\left(b_{\mu}^{(v)}\right)$ eine Folge mit gleichmässig begrenzten Zeilen und:

(94) $\varliminf_{\nu=\infty} \varliminf_{\mu=\infty} b_{\mu}^{(\nu)}=\lim _{\mu, \nu=\infty} b_{\mu}^{(\nu)}=\underline{b}, \varlimsup_{\nu=\infty} \varlimsup_{\mu=\infty} b_{\mu}^{(\nu)}=\varlimsup_{\mu, \nu=\infty} b_{\mu}^{(\nu)}=\bar{b}, \quad \underline{b}<\bar{b}$.

Bedeutet sodann $\left(c_{\mu}^{(\nu)}\right)$ eine Folge mit ungleichmässig begrensten Zeilen, für welche:

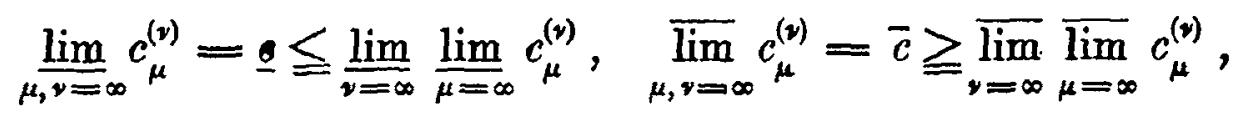

$$
\begin{aligned}
& \underline{b}<\underline{c} \leqq \bar{c}<\bar{b},
\end{aligned}
$$

so bilde man aus den Termen der beiden Doppelfolgen $\left(b_{\mu}^{(\nu)}\right),\left(c_{\mu}^{(\nu)}\right)$ eine einzige $\left(a_{\mu}^{(\nu)}\right)$ indem man setzt:

$$
a_{\mu}^{(2 v)}=b_{\mu}^{(v)}, \quad a_{\mu}^{(2 v+1)}=c_{\mu}^{(\nu)} \quad(\mu=0,1,2, \cdots, \nu=0,1,2, \cdots)
$$

(d. h. die Zeilen der Folge $\left(a_{\mu}^{(\nu)}\right)$ bestehen abwechselnd aus den Zeilen der beiden Folgen $\left(b_{\mu}^{(v)}\right),\left(c_{\mu}^{(v)}\right)$. Alsdann hat man offenbar:

$$
\varliminf_{\nu=\infty} \varliminf_{\mu=\infty} a_{\mu}^{(v)}=\varliminf_{\mu, \nu=\infty} a_{\mu}^{(\nu)}=\underline{b}, \quad \varlimsup_{\nu=\infty} \varlimsup_{\mu=\infty} a_{\mu}^{(\nu)}=\varlimsup_{\mu, v=\infty} a_{\mu}^{(\nu)}=\bar{b},
$$

obschon die.Zeilen der Folge $\left(a_{\mu}^{(v)}\right)$ nicht mehr gleichmässig begrenzt sind.

In analoger Weise kann man aus einer Folge mit einseitig gleichmässig begrenzten oder gleichmässig unbegrenzten Zeilen durch Einschaltung der Zeilen einer anderen Folge eine solehe bilden, welche jene Eigenschatit 
nicht mehr besitzt, während die in Frage kommenden Limites unverändert bleiben.

Hiergegen lassen sich die speciellen Sätze (II a), (IIb) in folgender Weise umkehren:

(III) Existirt $\lim _{\mu, v=\infty} a_{\mu}^{(\nu)}$ als endliche Zahl $A$, in welchem Falle dann (nach Satz (Ia) auch:

$$
\lim _{\nu=\infty} \varlimsup_{\mu=\infty} a_{\mu}^{(v)}=\lim _{\mu=\infty} \varlimsup_{v=\infty} a_{\mu}^{(v)}=A,
$$

so sind die Zeilen und Colonnen mit eventuellem Ausschluss einer endlichen Anzahl gleichmässig begrenat.

Beweis. Aus der Vorsussetzung $\lim _{\mu, v=\infty} a_{\mu}^{(v)}=A$ folgt zunächst, dass zu jedem $\varepsilon>0$ zwei natürliche Zahlen $m, n^{\prime}$ fixirt werden können, dass:

$$
A-\frac{\varepsilon}{2} \leqq a_{\mu}^{(\nu)} \leqq A+\frac{\varepsilon}{2} \quad \text { für: } \quad \mu \geqq m, \quad \nu \geqq n^{\prime} .
$$

Da sodann auch:

$$
\lim _{\nu=\infty} \varlimsup_{\mu=\infty} a_{\mu}^{(v)}=A
$$

so erkennt man, dass die $\prod_{\mu=\infty} a_{\mu}^{(\nu)}, \varlimsup_{\mu=\infty} a_{\mu}^{(v)}$ zum mindesten von einem bestimmten Index $\nu$ ab endliche Zahlen sein müssen, etwa:

und dass sodann:

$$
\varliminf_{\mu=\infty} a_{\mu}^{(\nu)}=\underline{a}^{(\nu)}, \quad \varlimsup_{\mu=\infty} a_{\mu}^{(\nu)}=\bar{a}^{(\nu)},
$$

$$
A-\frac{\varepsilon}{2} \leqq\left\{\frac{\underline{a}^{(v)}}{\bar{a}^{(v)}}\right\} \leqq A+\frac{\varepsilon}{2} \quad \text { etwa für: } \nu \geqq n^{\prime \prime} .
$$

Man hat also inśbesondere:

$$
a^{(*)}-\varepsilon \leqq A-\frac{\varepsilon}{2}, \quad A+\frac{\varepsilon}{2} \leqq \bar{a}^{(v)}+\varepsilon \quad \text { für: } \quad \nu \geqq n^{\prime \prime}
$$

und daher, wenn $m$ eine Zahl bedentet, die nicht kleiner als $n^{\prime}$ and $n^{\prime \prime}$, durch Verbindung der Ungleichungen (102) und (99):

$$
\underline{a}^{(v)}-\omega_{i}^{(\eta)} \leqq \bar{a}^{(v)}+\varepsilon \quad \text { für }: \mu \geq m ; \quad v \geqq n
$$

d. h. die Zeilen der Doppelfolge sind gleichmässig begrenst. Das analoge gilt dann wiederum für die Colonnen. -

(IIII) Besteht eine der drei Beciehungen:

$\lim _{\nu \rightarrow \infty} \prod_{\mu=\infty} x_{\mu}=+\infty ; \lim _{\mu=\infty} \varlimsup_{\nu=\infty} a_{\mu}^{(\nu)}=+\infty ; \lim _{\mu, v \rightarrow \infty} a_{\mu}^{(\nu)}=+\infty$,

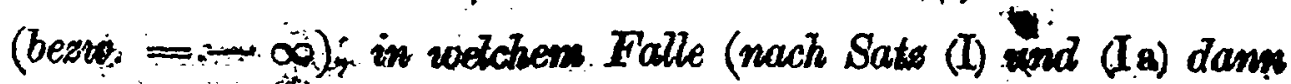
allemal and de beides anderen erfiul sind, so sind die 
Zeilen und Colonnen mit eventuellem Ausschluss einer endlichen Anzahl gleichmässig unbegrenzt.

Dieser letztere Satz bedarf keines weiteren Beweises. Da man nämlich, wie schon in seiner Fassung enthalten, allemal von vornherein $\lim _{\mu, \nu=\infty} a_{\mu}^{(\nu)}=+\infty$ bezw. $-\infty$ annehmen kann, so besteht die DivergenzBedingung (3a) (s. \$1), welche mit der Eigenschaft der ,gleichmässigen Unbegrenztheit" identisch ist (vgl. Fussn. 1), p. 316).

Durch Zusammenfassung der Sätze (IIa), (IIIa) bezw. (IIb), (IIIb) und Berücksichtigung von (Ia) gewinnt man schliesslich noch die folgenden allgemeinsten Sätze dieser Art:

(IVa) Für die Convergenz der Doppelfolge $\left(\alpha_{\mu}^{(\nu)}\right)$ ist nothwendig und hinreichend, dass die Zeilen oder Colonnen mit eventuellem Ausschluss einer endlichen Anzahl gleichmässig begrenzt sind und dass $\lim _{\nu=\infty} \varlimsup_{\mu=\infty} a_{\mu}^{(v)}$ bezw. $\lim _{\mu=\infty} \varlimsup_{\nu=\infty} a_{\mu}^{(v)}$ existirt. Ist diese Bedingung für die Zeilen und $\lim _{\nu=\infty} \varlimsup_{\mu=\infty} a_{\mu}^{(v)}$ erfüllt, so besteht sie auch für die Colonnen und $\lim _{\mu=\infty} \varlimsup_{\nu=\infty} a_{\mu}^{(\nu)}$, vice versa, und man hat dann allemal:

$$
\lim _{\mu, \nu=\infty} a_{\mu}^{(\nu)}=\lim _{\mu=\infty} \varlimsup_{\nu=\infty} a_{\mu .}^{(\nu)}=\lim _{\nu=\infty} \varlimsup_{\mu=\infty} a_{\mu}^{(\nu)} .
$$

(IVb) Für die eigentlieke Divergene der. Doppelfolge ist nothwendig und finreichend; dass die. Zeiten oder Colonnen mit eventuellem Ausschluss einer endichen Anzahl gleichmässig unbegrenzt sind̈. Ist diese Bedingung fïr die Zeilen erfüllt, so besteht sie auch für die Colonnen, vice versa; und man hat dann allemal:

$$
\lim _{\mu, \nu=\infty}=\lim _{\mu=\infty} \varlimsup_{i=\infty} a_{\mu}^{(v)}=\lim _{v=\infty} \varlimsup_{\mu=\infty} a_{\mu}^{(v)}=+\infty \text { bezw. }=-\infty \text {. }
$$

München, December 1896 und Juni 1899. 